\title{
Perspectives and obstacles for optimisation of brake pads with respect to stability criteria
}

\author{
O.N. Kirillov \\ Dynamics Group, \\ Department of Mechanical Engineering, \\ Technische Universität Darmstadt, \\ Hochschulstr. 1, 64289 Darmstadt, Germany \\ Fax: 496151164125 \\ E-mail: kirillov@dyn.tu-darmstadt.de
}

\begin{abstract}
Structural modifications of the brake components is an evident passive counter-measure directed on elimination of propensity of the brake to a squeal. The assignment of the unstable eigenvalues into the stable part of the complex plane by the structural optimisation of the non-conservative rotor system requires, however, careful investigation of the doublets in the Campbell diagram. Such an investigation substantially illuminates the perspectives and obstacles for the structural optimisation of brake pads with respect to stabilising eigenvalue assignment and provides explicit formulas, which allow one to assign desirable stable and unstable modes of the coupled system selectively.
\end{abstract}

Keywords: disk brake; rotor dynamics; frequency-speed diagram; brake pads; sensitivity analysis; optimisation; subcritical flutter; brake squeal.

Reference to this paper should be made as follows: Kirillov, O.N. (2009) 'Perspectives and obstacles for optimisation of brake pads with respect to stability criteria', Int. J. Vehicle Design, Vol. 51, Nos. 1/2, pp.143-167.

Biographical notes: Oleg N. Kirillov received his $\mathrm{PhD}$ in Theoretical Mechanics at the Moscow State Lomonosov University in Russia. He was a Visiting Scholar at the Technical University of Denmark, Dalian University of Technology, University of Hannover, and Research Center Dresden-Rossendorf. Currently he is a Research Scientist at the Department of Mechanical Engineering, Technical University of Darmstadt, Germany. His research interests include issues related to stability of non-conservative systems, gyroscopic stabilisation, rotor dynamics, structural optimisation, dissipation-induced instabilities and perturbation theory of non-self-adjoint operators. $\mathrm{He}$ is author of a great deal of research studies published at national and international peer-reviewed journals and conference proceedings. 


\section{Introduction}

The self-excited vibrations in brakes known as the brake squeal phenomenon is a challenging problem for the modern rotor dynamics (see e.g., recent overviews by Kinkaid et al. (2003), Ouyang et al. (2005), Chen et al. (2006) and Hoffmann and Gaul (2008)), because its "reliable reproduction or even prediction ... is still not possible" (Ostermeyer and Müller, 2008). Structural modification of the elements of a brake such as a rotor and pads is considered as an obvious passive counter-measure directed on elimination of propensity of the brake to a squeal, see, e.g., Abu Bakar et al. (2008), Penninger and Swift (2004) and Fieldhouse et al. (2004, 2008). The attempts to find the perfect brake are, however, still extensively based on the 'trial-and-error' method (Ostermeyer and Müller, 2008).

Some research works have examined simplified models of rotating elastic bodies of revolution in frictional contact and studied numerically the sensitivity of the onset of the self-excited vibrations with respect to the geometry of the friction pads. Xiong and Hutton (1994) have investigated a rotating circular string with point and distributed restraining springs and found that the arc length of the elastic support is one of the factors determining which crossings of the eigenvalue branches of the Campbell diagram (Campbell, 1924) veer away into avoided crossings. Despite the perturbed string remains stable in the subcritical rotational speed range, the observed highly selective effect of the geometry of the elastic support allows one to expect that with dissipative and non-conservative forces taken into account the structural modification of the pads similarly will most easily excite a particular mode in the vicinity of the locations of the double eigenvalues in the Campbell diagram. In a recent work, Kang et al. (2008) confirmed this expectation by demonstrating the selective properties of the contact span angle for the squeal onset in the models of a thin disk with finite frictional contact area. Experimental research of Fieldhouse et al. (2008) and Oura et al. (2008) revealed the sensitivity of the friction-induced instabilities to the distributed contact pressure and stiffness fluctuations.

The need for a rational design of the brake components as well as the inevitable uncertainties in the tribological properties of the pads makes it reasonable to look at the multiparameter stability analysis of a brake as at a non-conservative structural optimisation problem with respect to stability criteria. Then, the merit functional is the critical value of one of the crucial well-measurable parameters at the onset of squeal or even the eigenvalues themselves whereas the design variables are those describing the material and geometrical properties of the pads. We would like to know where the variations in the design should be made in order to influence the stability of a desirable vibration mode and to assign the real part of the corresponding eigenvalue to the left side of the complex plane.

In a recent work Ouyang (2008) revealed that it is quite difficult and sometimes even impossible to assign the real parts of eigenvalues in friction-induced vibration problems by means of mass, stiffness and damping modifications to stabilise the system. It is well-known, however, that the main obstacles in the problems of structural optimisation are that the merit functional is generally non-smooth with respect to the design variables (Seyranian et al., 1994); in non-conservative problems the functional is additionally non-convex (Kirillov and Seyranian, 1998). The singular points of the functional, frequently related to multiple eigenvalues, complicate considerably the geometry of the stability domain. Therefore, the sensitivity analysis of the multiple 
eigenvalues as well as the analysis of the corresponding singular points of the stability frontier is by necessity required for the correct solving structural optimisation problems in the non-conservative systems possessing friction-induced vibrations.

\section{Disk brake as an axially symmetric rotor with an anisotropic stator}

Linear stability analysis is widely used to predict possible instabilities in the dynamics of rotor systems to which the disk brake can be attributed. The presence of imperfections in rotor and stator makes the operator of the linearised equations of motion non-self-adjoint with time-dependent coefficients, which considerably complicates the stability analysis (Lee et al., 2007). Nevertheless, an axially symmetric rotor with an anisotropic stator as well as an asymmetric rotor with an isotropic stator can be described as an autonomous non-conservative gyroscopic system (Genta, 2007). Unless the vented disk or the disk with specially manufactured symmetry-breaking pattern (Fieldhouse et al., 2004) is considered, the model of an axially symmetric rotor with an anisotropic stator is reasonable for the description of the disk brake as well as for the description of other sound-emitting rotating elastic bodies of revolution in frictional contact such as the drum brake or the glass harmonica (Kirillov, 2008a).

When the brake pads are not applied, the unperturbed system is assumed to be isotropic with the constant speed of the rotor $\Omega$. It is described by the equations of a standard non-dimensional form (Genta, 2007)

$$
\left(\begin{array}{cc}
\widetilde{\mathbf{M}} & \mathbf{0} \\
\mathbf{0} & \widetilde{\mathbf{M}}
\end{array}\right) \ddot{\mathbf{z}}+2 \Omega\left(\begin{array}{cc}
\mathbf{0} & -\widetilde{\mathbf{G}} \\
\widetilde{\mathbf{G}} & \mathbf{0}
\end{array}\right) \dot{\mathbf{z}}+\left[\left(\begin{array}{cc}
\widetilde{\mathbf{P}} & \mathbf{0} \\
\mathbf{0} & \widetilde{\mathbf{P}}
\end{array}\right)+\Omega^{2}\left(\begin{array}{cc}
\widetilde{\mathbf{G}}^{2}+\widetilde{\mathbf{C}} & \mathbf{0} \\
\mathbf{0} & \widetilde{\mathbf{G}}^{2}+\widetilde{\mathbf{C}}
\end{array}\right)\right] \mathbf{z}=0
$$

with $\mathbf{z}=\mathbb{R}^{2 n}$ and $\widetilde{\mathbf{M}}=\operatorname{diag}(1,1, \ldots, 1), \widetilde{\mathbf{G}}=\operatorname{diag}(1,2, \ldots, n), \widetilde{\mathbf{P}}=\operatorname{diag}\left(\omega_{1}^{2}, \omega_{2}^{2}, \ldots\right.$, $\left.\omega_{n}^{2}\right)$, and $\widetilde{\mathbf{C}}=\operatorname{diag}\left(c_{1}^{2}, c_{2}^{2}, \ldots, c_{n}^{2}\right)$, see also Spelsberg-Korspeter et al. (2009). Equation (1) generalises the two-dimensional setting considered by Kirillov (2008b).

At standstill the eigenvalues of the system $i \omega_{s}$ are double semi-simple with two linearly independent eigenvectors. The distribution of the doublets $\omega_{s}$ as a function of the integer index $s$ is usually different for various bodies of revolution. For example, $\omega_{s}=s$ corresponds to the natural frequency $f_{s}=\frac{s}{2 \pi r} \sqrt{\frac{P}{\rho}}$ of a circular string of radius $r$, circumferential tension $P$ and mass density $\rho$ per unit length (Xiong and Hutton, 1994). The natural frequency of the doublet mode with one nodal circle and $s$ nodal diameters $f_{s}=\frac{\omega_{s}^{2} h}{2 \pi a^{2}} \sqrt{\frac{E}{12 \rho\left(1-\nu^{2}\right)}}$ of the clamped-free annular disk of thickness $h$, inner radius $b$, outer radius $a$, mass per unit volume $\rho$, Poisson's ratio $\nu$, and elastic modulus $E$ corresponds to the non-dimensional eigenvalue constant $\omega_{s}$, which can be found numerically for a given Poisson's ratio and radius ratio $b / a$. According to Gabrielson (1999) for $\nu=0.3$ and $b / a=0.1$ the first two constants are $\omega_{1}=5.2605$, and $\omega_{2}=6.0779$. Notice that in experiments squeal noise often associates with the rotor vibrating modes having just nonzero number of nodal diameters (Fieldhouse and Beveridge, 2000).

In the following we prefer to consider equation (1) in a new equivalent form that shows the doublets explicitly

$$
\ddot{\mathbf{x}}+2 \Omega \mathbf{G} \dot{\mathbf{x}}+\left(\mathbf{P}+\Omega^{2}\left(\mathbf{G}^{2}+\mathbf{C}\right)\right) \mathbf{x}=0,
$$


where $\mathbf{x}=\mathbb{R}^{2 n}, \mathbf{P}=\operatorname{diag}\left(\omega_{1}^{2}, \omega_{1}^{2}, \omega_{2}^{2}, \omega_{2}^{2}, \ldots, \omega_{n}^{2}, \omega_{n}^{2}\right)=\mathbf{P}^{T}$ is the stiffness matrix of the rotor, $\mathbf{C}=\operatorname{diag}\left(c_{1}^{2}, c_{1}^{2}, c_{2}^{2}, c_{2}^{2}, \ldots, c_{n}^{2}, c_{n}^{2}\right)$ is the matrix of the centrifugal stiffness, and $\mathbf{G}=-\mathbf{G}^{T}$ is the matrix of gyroscopic forces defined as

$$
\mathbf{G}=\operatorname{blockdiag}(\mathbf{J}, 2 \mathbf{J}, \ldots, n \mathbf{J}), \quad \mathbf{J}=\left(\begin{array}{cc}
0 & -1 \\
1 & 0
\end{array}\right) .
$$

Separating time by the substitution $\mathbf{x}=\mathbf{u} \exp (\lambda t)$ into equation (2), we arrive at the eigenvalue problem for the matrix operator $\mathbf{L}_{0}$

$$
\mathbf{L}_{0}(\Omega) \mathbf{u}:=\left(\mathbf{I} \lambda^{2}+2 \Omega \mathbf{G} \lambda+\mathbf{P}+\Omega^{2}\left(\mathbf{G}^{2}+\mathbf{C}\right)\right) \mathbf{u}=0 .
$$

As a consequence of the block-diagonal structure of the sparse matrices $\mathbf{G}$ and $\mathbf{P}$, the eigenvalues of $\mathbf{L}_{0}$ are found explicitly as

$$
\lambda_{s}^{ \pm}=i \sqrt{\omega_{s}^{2}+c_{s}^{2} \Omega^{2}} \pm i \Omega s, \quad \overline{\lambda_{s}^{ \pm}}=-i \sqrt{\omega_{s}^{2}+c_{s}^{2} \Omega^{2}} \mp i \Omega s,
$$

where bar over a symbol denotes complex conjugate. Rotation causes the doublet modes $\pm i \omega_{s}$ to split. The newborn pair of simple eigenvalues $\lambda_{s}^{ \pm}$corresponds to the forward and backward travelling waves, which propagate along the circumferential direction. Viewed from the stationary frame, the frequency of the forward travelling wave appears to increase and that of the backward travelling wave appears to decrease, as the spin increases. Double eigenvalues thus originate again at non-zero angular velocities, forming the nodes of the spectral mesh (Kirillov, 2008b) of the crossed frequency curves in the Campbell diagram in the plane 'frequency' vs. 'angular velocity'.

At the angular velocity

$$
\Omega_{s}^{c r}=\frac{\omega_{s}}{\sqrt{s^{2}-c_{s}^{2}}}
$$

the frequency of the $s$ th backward travelling wave vanishes to zero $\left(\lambda_{s}^{ \pm}=\overline{\lambda_{s}^{ \pm}}=\right.$ 0 ), so that the wave remains stationary in the non-rotating frame. Campbell (1924) calls these velocities critical whereas Genta (2007) refers to them as the thresholds of (divergence) instability. We define the lowest one of such velocities as critical and denote it $\Omega_{c r}$. When the speed of rotation exceeds the critical speed, some backward waves, corresponding to the eigenvalues $\overline{\lambda_{s}^{ \pm}}$, travel slower than the disk rotation speed and appear to be travelling forward (reflected waves). The effective energy of the reflected wave is negative and that of the forward and backward travelling waves is positive. Therefore, in the subcritical speed region $|\Omega|<\Omega_{c r}$ all the crossings of the frequency curves correspond to the forward and backward modes of the same signature, while in the supercritical speed region $|\Omega|>\Omega_{c r}$ there exist crossings that are formed by the reflected and forward/backward modes of opposite signature. As a consequence of the fundamental Krein's theorem (MacKay, 1986), under perturbations preserving the Hamiltonian structure of the equations of motion the crossings in the subcritical region veer away into avoided crossings (stability), while in the supercritical region the crossings with the mixed signature turn into the rings of complex eigenvalues - bubbles of instability (MacKay, 1986) - leading to 
flutter known also as the 'mass and stiffness instabilities' (Mottershead, 1998) or as the "resonance with the static force applied in a direction perpendicular to the disk rotation plane" (Genta, 2007).

A supercritical flutter frequently occurs in the high speed applications like turbines, circular saws, and computer storage devices, while the subcritical flutter - either desirable as a source of instability at low speeds as in the case of musical instruments like the singing wine glass and a glass harmonica or undesirable as in the case of the squealing disk- and drum brakes - is an elusive phenomenon.

In the following, using perturbation theory of multiple eigenvalues we show that even if the eigenvalue branches in the subcritical region are well separated at the avoided crossings, created by the stiffness variation of the stator, they can be forced to bend with the origination of arcs of complex eigenvalues with positive real parts by the indefinite damping, which comes to the equations of motion, e.g., from the negative friction-velocity gradient (Hagedorn, 1988; Spurr, 1961). In the space of the gyroscopic, damping, and stiffness parameters the zones of the subcritical dissipation-induced flutter turn out to be cones with the apexes at the points, corresponding to the nodes of the spectral mesh. We show that the orientation of the cone is substantially determined by the structure of the damping matrix, which can be chosen in such a way that the system is unstable even for significant magnitudes of the stiffness matrix detuning. The conical zones of the subcritical flutter bifurcate into the geometrically more complicated domains having couples of Whitney's umbrella singularities when non-conservative positional forces (originated from the follower forces or from the moment generated by variation in friction forces, see Ouyang et al. (2005), Chen et al. (2006) and Kang et al. (2008)) are additionally taken into account. We describe in detail this process and discuss how the consequences of this nontrivial geometry of the space of the design parameters complicates the search for an optimal structural modification of the brake pads. We will explicitly demonstrate how to navigate through the singularities in the parameter space on the examples of the modification of the stiffness, damping and non-conservative matrices leading to the assignment of stable and unstable eigenvalues corresponding to the desirable vibration modes.

\section{Sensitivity analysis of the doublet modes}

The equations of motion of the disk brake with the applied friction pads can be considered as a perturbation of the system (2), which yields the variation of the stiffness, damping, and non-conservative positional (circulatory) terms

$$
\ddot{\mathbf{x}}+(2 \Omega \mathbf{G}+\delta \mathbf{D}) \dot{\mathbf{x}}+\left(\mathbf{P}+\Omega^{2}\left(\mathbf{G}^{2}+\mathbf{C}\right)+\kappa \mathbf{K}+\nu \mathbf{N}\right) \mathbf{x}=0,
$$

where the damping, $\mathbf{D}$, stiffness, $\mathbf{K}$, and non-conservative, $\mathbf{N}$, matrices can depend on $\Omega$. The intensity of the perturbation is controlled by the factors $\delta, \kappa$ and $\nu$. For a given structure of the matrices, stability depends on the correctly chosen combination of the parameters (Chevillot et al., 2008; Kirillov, 2008b). When the intensities $\delta, \kappa$, and $\nu$ are fixed, one affects the eigenvalue assignment by modifying the structure of the corresponding matrices (see, e.g., Ouyang, 2008).

For simplicity we restrict our subsequent consideration by $2 n=4$ degrees of freedom and neglect the centrifugal stiffening, $\mathbf{C}$, in equation (6). Then, we can choose 
the eigenvectors $\mathbf{u}_{s}^{+}$, corresponding to the eigenvalues $\lambda_{s}^{+}=i \omega_{s}+i s \Omega$ and $\overline{\lambda_{s}^{-}}=$ $-i \omega_{s}+i s \Omega$ of the unperturbed isotropic rotor system as

$$
\mathbf{u}_{1}^{+}=(-i, 1,0,0)^{T}, \quad \mathbf{u}_{2}^{+}=(0,0,-i, 1)^{T},
$$

where $i=\sqrt{-1}$, and the eigenvectors $\mathbf{u}_{s}^{-}$, corresponding to the eigenvalues $\lambda_{s}^{-}=i \omega_{s}-i s \Omega$ and $\overline{\lambda_{s}^{+}}=-i \omega_{s}-i s \Omega$, as

$$
\mathbf{u}_{1}^{-}=(i, 1,0,0)^{T}, \quad \mathbf{u}_{2}^{-}=(0,0, i, 1)^{T} .
$$

Consider a double semi-simple eigenvalue $\lambda_{0}$ at the crossing of the eigenvalue branches $\lambda_{s}^{\varepsilon}=i \alpha \omega_{s}+i \varepsilon s \Omega$ and $\lambda_{t}^{\sigma}=i \beta \omega_{t}+i \sigma t \Omega$, where $\quad \alpha, \beta, \varepsilon, \sigma= \pm 1$. Denote the corresponding eigenvectors $\mathbf{u}_{s}^{\varepsilon}$ and $\mathbf{u}_{t}^{\sigma}$.

The double eigenvalue $\lambda_{0}$ and the value of the gyroscopic parameter $\Omega_{0}$ at the crossing are

$$
\Omega_{0}=\frac{\alpha \omega_{s}-\beta \omega_{t}}{\sigma t-\varepsilon s}, \quad \lambda_{0}=i \frac{\alpha \sigma \omega_{s} t-\beta \varepsilon \omega_{t} s}{\sigma t-\varepsilon s}=i \omega_{0},
$$

and the following useful relations hold

$$
\lambda_{0}-\varepsilon i s \Omega_{0}=i \alpha \omega_{s}, \quad \lambda_{0}-\sigma i t \Omega_{0}=i \beta \omega_{t} .
$$

Let $\mathbf{M}$ be one of the perturbing matrices $\mathbf{D}, \mathbf{K}$ or $\mathbf{N}$. In the following we will use the decomposition of the matrix $\mathbf{M} \in \mathbb{R}^{4 \times 4}$ into the blocks $\mathbf{M}_{s t} \in \mathbb{R}^{2 \times 2}$

$$
\mathbf{M}=\left(\begin{array}{ll}
\mathbf{M}_{11} & \mathbf{M}_{12} \\
\mathbf{M}_{21} & \mathbf{M}_{22}
\end{array}\right), \quad \mathbf{M}_{s t}=\left(\begin{array}{cc}
m_{2 s-1,2 t-1} & m_{2 s-1,2 t} \\
m_{2 s, 2 t-1} & m_{2 s, 2 t}
\end{array}\right) .
$$

Thus, $\mathbf{D}_{s t}=\mathbf{D}_{t s}^{T}, \mathbf{K}_{s t}=\mathbf{K}_{t s}^{T}$, and $\mathbf{N}_{s t}=-\mathbf{N}_{t s}^{T}$.

We consider a general perturbation of the matrix operator of the isotropic rotor $\mathbf{L}_{0}(\Omega)+\Delta \mathbf{L}(\Omega)$. The size of the perturbation $\Delta \mathbf{L}(\Omega)=\delta \lambda \mathbf{D}+\kappa \mathbf{K}+\nu \mathbf{N} \sim \varepsilon$ is small, where $\varepsilon=\left\|\Delta \mathbf{L}\left(\Omega_{0}\right)\right\|$ is the Frobenius norm of the perturbation at $\Omega=\Omega_{0}$. For small $\Omega$ and $\varepsilon$ perturbation of the double semi-simple eigenvalue $\lambda_{0}=i \omega_{0}$ with the eigenvectors $\mathbf{u}_{s}^{\varepsilon}$ and $\mathbf{u}_{t}^{\sigma}$ at $\Omega=\Omega_{0}$, is given by the formula

$$
\operatorname{det}\left(\mathbf{F}+\left(\lambda-\lambda_{0}\right) \mathbf{H}\right)=0,
$$

where the entries of the matrices $\mathbf{H}$ and $\mathbf{F}$ are

$$
\begin{aligned}
H_{s t}^{\varepsilon \sigma}= & 2 i \omega_{0}\left(\overline{\mathbf{u}}_{s}^{\varepsilon}\right)^{T} \mathbf{u}_{t}^{\sigma}+2 \Omega_{0}\left(\overline{\mathbf{u}}_{s}^{\varepsilon}\right)^{T} \mathbf{G} \mathbf{u}_{t}^{\sigma}, \\
F_{s t}^{\varepsilon \sigma}= & \left(2 i \omega_{0}\left(\overline{\mathbf{u}}_{s}^{\varepsilon}\right)^{T} \mathbf{G} \mathbf{u}_{t}^{\sigma}+2 \Omega_{0}\left(\overline{\mathbf{u}}_{s}^{\varepsilon}\right)^{T} \mathbf{G}^{2} \mathbf{u}_{t}^{\sigma}\right)\left(\Omega-\Omega_{0}\right) \\
& +i \omega_{0}\left(\overline{\mathbf{u}}_{s}^{\varepsilon}\right)^{T} \mathbf{D} \mathbf{u}_{t}^{\sigma} \delta+\left(\overline{\mathbf{u}}_{s}^{\varepsilon}\right)^{T} \mathbf{K} \mathbf{u}_{t}^{\sigma} \kappa+\left(\overline{\mathbf{u}}_{s}^{\varepsilon}\right)^{T} \mathbf{N} \mathbf{u}_{t}^{\sigma} \nu .
\end{aligned}
$$

When $\delta=0, \nu=0$, and $\kappa=0$, the non-diagonal terms of the matrices $\mathbf{H}$ and $\mathbf{F}$ are zero, while the diagonal ones with the use of the relations (10) take the form

$$
\begin{aligned}
H_{s s}^{\varepsilon \varepsilon} & =4 i \alpha \omega_{s}, \quad F_{s s}^{\varepsilon \varepsilon}=4 s \varepsilon \alpha \omega_{s}\left(\Omega-\Omega_{0}\right), \\
H_{t t}^{\sigma \sigma} & =4 i \beta \omega_{t}, \quad F_{t t}^{\sigma \sigma}=4 t \sigma \beta \omega_{t}\left(\Omega-\Omega_{0}\right) .
\end{aligned}
$$


Therefore, at the crossing (9) the perturbation formula (12) describes correctly the eigenvalue branches: $\Delta \lambda=i s \Delta \Omega \varepsilon$ and $\Delta \lambda=i t \Delta \Omega \sigma$, where $\varepsilon, \sigma= \pm 1$.

Owing to the relative simplicity of the matrices of the unperturbed system and therefore its eigenvectors, we can calculate the scalar products in equation (14) and express them by means of the entries $d_{j k}, k_{j k}$, and $n_{j k}$ of the damping, stiffness, and non-conservative matrices

$$
\begin{aligned}
& \left(\overline{\mathbf{u}}_{s}^{\varepsilon}\right)^{T} \mathbf{D} \mathbf{u}_{t}^{\sigma}=\sigma \operatorname{tr} \mathbf{D}_{s t} \mathbf{I}_{\varepsilon \sigma}+i \operatorname{tr} \mathbf{D}_{s t} \mathbf{J}_{\varepsilon \sigma} \\
& \left(\overline{\mathbf{u}}_{s}^{\varepsilon}\right)^{T} \mathbf{K} \mathbf{u}_{t}^{\sigma}=\sigma \operatorname{tr} \mathbf{K}_{s t} \mathbf{I}_{\varepsilon \sigma}+i \operatorname{tr} \mathbf{K}_{s t} \mathbf{J}_{\varepsilon \sigma} \\
& \left(\overline{\mathbf{u}}_{s}^{\varepsilon}\right)^{T} \mathbf{N u}_{t}^{\sigma}=\sigma \operatorname{tr} \mathbf{N}_{s t} \mathbf{I}_{\varepsilon \sigma}+i \operatorname{tr} \mathbf{N}_{s t} \mathbf{J}_{\varepsilon \sigma}
\end{aligned}
$$

where the auxiliary $2 \times 2$ matrices used for the compactness of the notation are

$$
\mathbf{I}_{\varepsilon \sigma}=\left(\begin{array}{cc}
\varepsilon & 0 \\
0 & \sigma
\end{array}\right), \quad \mathbf{J}_{\varepsilon \sigma}=\left(\begin{array}{cc}
0 & -\sigma \\
\varepsilon & 0
\end{array}\right)
$$

With the coefficients (13)-(19) the sensitivity of the doublet $\lambda_{0}$ at the crossing (9) of the Campbell diagram is described by the expression, which generalises the result of Kirillov (2008b) obtained for the case of two degrees of freedom,

$$
\Delta \lambda=i \frac{\Delta \Omega}{2}(s \varepsilon+t \sigma)+\frac{i}{8}\left(\frac{A_{1}}{\alpha \omega_{s}}+\frac{B_{1}}{\beta \omega_{t}}\right) \pm i \sqrt{c},
$$

where

$$
c=\left[\frac{\Delta \Omega}{2}(s \varepsilon-t \sigma)+\frac{1}{8}\left(\frac{A_{1}}{\alpha \omega_{s}}-\frac{B_{1}}{\beta \omega_{t}}\right)\right]^{2}-\frac{A_{2}^{2}+B_{2}^{2}}{16 \alpha \beta \omega_{s} \omega_{t}},
$$

and

$$
\begin{aligned}
& A_{1}=\delta \lambda_{0} \operatorname{tr} \mathbf{D}_{s s}+\kappa \operatorname{tr} \mathbf{K}_{s s}+\varepsilon 2 i \nu n_{2 s-1,2 s}, \\
& B_{1}=\delta \lambda_{0} \operatorname{tr} \mathbf{D}_{t t}+\kappa \operatorname{tr} \mathbf{K}_{t t}+\sigma 2 i \nu n_{2 t-1,2 t}, \\
& A_{2}=\sigma \nu \operatorname{tr} \mathbf{N}_{s t} \mathbf{I}_{\varepsilon \sigma}+i\left(\delta \lambda_{0} \operatorname{tr} \mathbf{D}_{s t} \mathbf{J}_{\varepsilon \sigma}+\kappa \operatorname{tr} \mathbf{K}_{s t} \mathbf{J}_{\varepsilon \sigma}\right), \\
& B_{2}=\sigma \nu \operatorname{tr} \mathbf{N}_{s t} \mathbf{J}_{\varepsilon \sigma}-i\left(\delta \lambda_{0} \operatorname{tr} \mathbf{D}_{s t} \mathbf{I}_{\varepsilon \sigma}+\kappa \operatorname{tr} \mathbf{K}_{s t} \mathbf{I}_{\varepsilon \sigma}\right) .
\end{aligned}
$$

It is remarkable that the formulas (21)-(23) describe sensitivity of the Campbell diagram with respect to the structural modification of the stator in the vicinity of the doublets in terms of the elements of the four block matrices decomposing the original operators of damping, stiffness, and non-conservative positional forces. This means that one can substantially affect the eigenvalue assignment varying relatively small number of parameters in the original matrices! In the following sections we will explicitely demonstrate this effect. 


\section{Stiffness modifications}

First we examine modifications of the stiffness matrix only. The Campbell diagram near the crossing of the eigenvalue branches $\lambda_{s}^{\varepsilon}=i \alpha \omega_{s}+i \varepsilon s \Omega$ and $\lambda_{t}^{\sigma}=i \beta \omega_{t}+i \sigma t \Omega$ of the unperturbed system is then approximately

$$
\Delta \lambda=i\left(\frac{\kappa}{8}\left(\alpha \frac{\operatorname{tr} \mathbf{K}_{s s}}{\omega_{s}}+\beta \frac{\operatorname{tr} \mathbf{K}_{t t}}{\omega_{t}}\right)+\frac{\Delta \Omega}{2}(s \varepsilon+t \sigma)\right) \pm i \sqrt{c_{\kappa}},
$$

with

$$
c_{\kappa}=\left(\frac{\kappa}{8}\left(\frac{\operatorname{tr} \mathbf{K}_{s s}}{\alpha \omega_{s}}-\frac{\operatorname{tr} \mathbf{K}_{t t}}{\beta \omega_{t}}\right)+\frac{\Delta \Omega}{2}(s \varepsilon-t \sigma)\right)^{2}+\frac{\kappa^{2}}{16} \frac{\left(\operatorname{tr} \mathbf{K}_{s t} \mathbf{I}_{\varepsilon \sigma}\right)^{2}+\left(\operatorname{tr} \mathbf{K}_{s t} \mathbf{J}_{\varepsilon \sigma}\right)^{2}}{\alpha \beta \omega_{s} \omega_{t}} .
$$

When the radicand $c_{\kappa}$ is positive (which is always fulfilled for $\alpha \beta>0$ ), the eigenvalues remain purely imaginary. For negative values of $c_{\kappa}$ the real parts of the eigenvalues can develop, yielding a non-trivial decay rate plot in addition to the Campbell diagram. The real parts are positive inside the sector in the $(\Omega, \kappa)$-plane bounded by the stability frontiers, which in the first approximation are straight lines $c_{\kappa}=0$

$$
\kappa=\frac{4(s \varepsilon-t \sigma)\left(\Omega-\Omega_{0}\right)}{\left(\begin{array}{c}
\frac{k_{2 t-1,2 t-1}+k_{2 t, 2 t}}{\beta \omega_{t}}-\frac{k_{2 s-1,2 s-1}+k_{2 s, 2 s}}{\alpha \omega_{s}} \\
\pm 2 \sqrt{\frac{\left(\varepsilon k_{2 s-1,2 t-1}+\sigma k_{2 s, 2 t}\right)^{2}+\left(\varepsilon k_{2 s-1,2 t}-\sigma k_{2 s, 2 t-1}\right)^{2}}{-\alpha \beta \omega_{s} \omega_{t}}}
\end{array}\right) .}
$$

Using formula (26) we now demonstrate the selective properties of the stiffness matrix modification. As a model problem we consider a rotor system with two doublets at standstill chosen to be $\omega_{1}=5$ and $\omega_{2}=6$ for simplicity of analytical expressions; from the other hand they mimic the first two eigenvalue constants of the clamped-free annular disk with one nodal circle and one and two nodal diameters (Gabrielson, 1999).

For positive $\Omega$ and $\kappa$ the Campbell diagram of the isotropic rotor possesses two modal critical speeds $\Omega_{1}^{c r}=3$ and $\Omega_{2}^{c r}=5$ with double zero eigenvalues. Substituting $\varepsilon=-\sigma=-1, \alpha=-\beta=1$ and $s=t=2$ into equation (26) we find the stability frontiers corresponding to the first modal critical speed, $\Omega_{1}^{c r}=3$,

$$
\kappa=\frac{8 \omega_{2}(\Omega-3)}{k_{33}+k_{44} \mp \sqrt{\left(k_{33}-k_{44}\right)^{2}+\left(k_{34}+k_{43}\right)^{2}}} .
$$

For the second modal critical speed, $\Omega_{2}^{c r}=5$, we have $s=t=1$ and $\varepsilon=-\sigma=-1$, $\alpha=-\beta=1$, which yields

$$
\kappa=\frac{4 \omega_{1}(\Omega-5)}{k_{11}+k_{22} \mp \sqrt{\left(k_{11}-k_{22}\right)^{2}+\left(k_{12}+k_{21}\right)^{2}}} .
$$

Expressions (27) and (28) describe the boundaries of the divergence instability domains corresponding to the positive real eigenvalues. 
The only crossing with the non-zero double eigenvalue that exists in the supercritical speed range $\Omega>3$

$$
\left(\Omega_{0}=11 / 3, \omega_{0}=4 / 3\right) \text { for } s=1, t=2, \varepsilon=-\sigma=-1, \alpha=-\beta=1,
$$

yields the flutter domain in the $(\Omega, \kappa)$-plane with the stability frontiers

$$
\kappa=\frac{12(\Omega-11 / 3)}{\frac{k_{11}+k_{22}}{\omega_{1}}+\frac{k_{33}+k_{44}}{\omega_{2}} \mp 2 \sqrt{\frac{\left(k_{13}-k_{24}\right)^{2}+\left(k_{14}+k_{23}\right)^{2}}{\omega_{1} \omega_{2}}}} .
$$

In the subcritical range $\Omega<3$ there exist only four crossings of the eigenvalue branches, whose locations in the Campbell diagram are

$$
\begin{array}{ll}
\left(\Omega_{0}=0, \omega_{0}=5\right) & \text { for } s=1, t=1, \varepsilon=-\sigma=1, \alpha=\beta=1, \\
\left(\Omega_{0}=0, \omega_{0}=6\right) & \text { for } s=2, t=2, \varepsilon=-\sigma=1, \alpha=\beta=1, \\
\left(\Omega_{0}=1 / 3, \omega_{0}=16 / 3\right) & \text { for } s=1, t=2, \varepsilon=-\sigma=1, \alpha=\beta=1, \\
\left(\Omega_{0}=1, \omega_{0}=4\right) & \text { for } s=1, t=2, \varepsilon=\sigma=-1, \alpha=\beta=1 .
\end{array}
$$

Figure 1(a) shows the Campbell diagram of the isotropic rotor with all the above mentioned seven types of possible crossings. The four subcritical crossings of the Campbell diagram of the isotropic rotor can only veer away due to the stiffness matrix modification. The largest spectral gap between the eigenvalue branches at $\Delta \Omega=0$ is given by the expression $2 \sqrt{c_{\kappa}}$, where the constant $c_{\kappa}$ for each of the four crossings listed in (31) is

$$
\begin{aligned}
& (0,5): c_{\kappa}=\frac{\kappa^{2}}{16 \omega_{1}^{2}}\left[\left(k_{11}-k_{22}\right)^{2}+\left(k_{12}+k_{21}\right)^{2}\right] \\
& (0,6): c_{\kappa}=\frac{\kappa^{2}}{16 \omega_{2}^{2}}\left[\left(k_{33}-k_{44}\right)^{2}+\left(k_{34}+k_{43}\right)^{2}\right] \\
& \left(\frac{1}{3}, \frac{16}{3}\right): c_{\kappa}=\frac{\kappa^{2}}{64}\left(\frac{k_{11}+k_{22}}{\omega_{1}}-\frac{k_{33}+k_{44}}{\omega_{2}}\right)^{2}+\frac{\kappa^{2}}{16} \frac{\left(k_{13}-k_{24}\right)^{2}+\left(k_{14}+k_{23}\right)^{2}}{\omega_{1} \omega_{2}} \\
& (1,4): c_{\kappa}=\frac{\kappa^{2}}{64}\left(\frac{k_{11}+k_{22}}{\omega_{1}}-\frac{k_{33}+k_{44}}{\omega_{2}}\right)^{2}+\frac{\kappa^{2}}{16} \frac{\left(k_{13}+k_{24}\right)^{2}+\left(k_{14}-k_{23}\right)^{2}}{\omega_{1} \omega_{2}} .
\end{aligned}
$$

The formulas (27), (28), (30) and (32) demonstrate that the crossings situated on the lines $\Omega=0$ and $\operatorname{Im} \lambda=0$ are influenced only by the entries of one of the two diagonal $2 \times 2$ subblocks of the stiffness perturbation matrix $\mathbf{K}$. Moreover, the pairs of the crossings $(0,5)$ and $(5,0)$ as well as $(0,6)$ and $(3,0)$ respond coherently to the variation of the very same entries - the effect found earlier by Xiong and Hutton (1994) in the rotating circular string with the partial elastic support. All the other crossings respond also to the changes in the entries of the two - anti-diagonal - subblocks of the matrix $\mathbf{K}$. 
Figure 1 (a) Campbell diagram of the isotropic rotor; (b)-(d) numerical calculations showing the veering and merging of modes, which were selectively caused by the modification of the stiffness matrix $\mathbf{K}$ with $\kappa=1.5$; (e) and (f) red regions are linear approximations (30) and (28) to the supercritical flutter and divergence domains corresponding to the diagrams (c) and (d), respectively (see online version for colours)

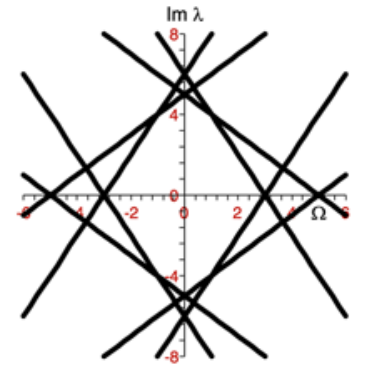

(a)

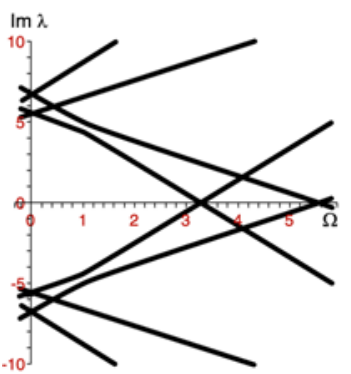

(b)

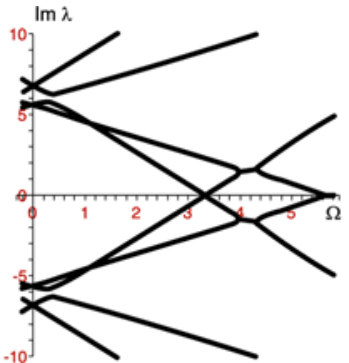

(c)

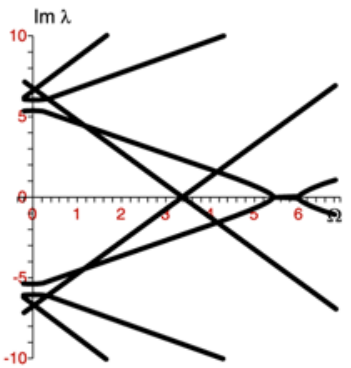

(d)

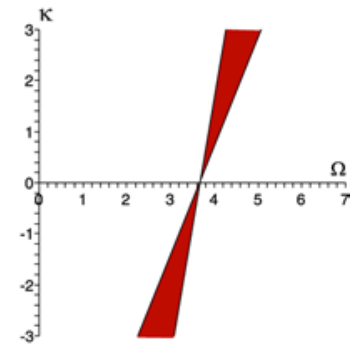

(e)

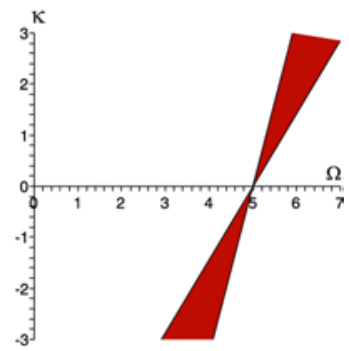

(f)

To illustrate the consequences of the performed sensitivity analysis we construct three different stiffness modification matrices with the use of the formulas (27), (28), (30) and (32)

$$
\mathbf{K}_{1}=\left(\begin{array}{cccc}
5 & 0 & 1 & 2 \\
0 & 5 & -2 & 1 \\
1 & -2 & 6 & 0 \\
2 & 1 & 0 & 6
\end{array}\right), \quad \mathbf{K}_{2}=\left(\begin{array}{cccc}
5 & 0 & 1 & 2 \\
0 & 5 & 2 & -1 \\
1 & 2 & 6 & 0 \\
2 & -1 & 0 & 6
\end{array}\right), \quad \mathbf{K}_{3}=\left(\begin{array}{cccc}
5 & 2 & 0 & 0 \\
2 & 5 & 0 & 0 \\
0 & 0 & 6 & 0 \\
0 & 0 & 0 & 6
\end{array}\right)
$$

Then, we numerically calculate the spectrum of the eigenvalue problem corresponding to the equation (6) with $\mathbf{K}=\mathbf{K}_{1}, \mathbf{K}=\mathbf{K}_{2}$, and $\mathbf{K}=\mathbf{K}_{3}$ for the same $\kappa=1.5$.

Figure 1(b) shows that the matrix $\mathbf{K}_{1}$ selectively affects only the subcritical crossing $(1,4)$, which veers away into the avoided crossing in the $(\Omega, \operatorname{Im} \lambda)$-plane. Eigenvalues at all the remaining crossings are non-distinguishable from the double ones within the precision of the used numerical method. The reason is that with the matrix $\mathbf{K}_{1}$ the radicands in the expressions (27), (28) and (30) as well as in the first three expressions (32) are zero, which means non-splitting in the first approximation. According to the last of the expressions (32), the only non-zero spectral gap corresponds to the crossing $(1,4)$. 
With the matrix $\mathbf{K}_{2}$ only the equation (30) and the third of equation (32) yield a non-trivial splitting at the crossings $(11 / 3,4 / 3)$ and $(1 / 3,16 / 3)$, respectively. This is confirmed by the numerical calculations shown in Figure 1(c). The matrix $\mathbf{K}_{3}$ possesses non-trivial splitting only in the equation (28) and in the first of the equation (32), corresponding to the crossings $(0,5)$ and $(5,0)$, which is in agreement with the numerical results of Figure 1(d). From Figure 1(e) and (f) it is clear that the linear approximations to the flutter and divergence instability domains (30) and (28) are in a very good qualitative and quantitative agreement with the numerically computed Campbell diagrams of Figure 1(c) and (d).

As it is seen from the numerical computations, the Campbell diagram of the perturbed isotropic rotor is essentially changed in the vicinity of the locations of the doublets of the unperturbed rotor. These loci are the true 'centres of control', because the main qualitative changes of the whole Campbell diagram can be effectively predicted by the sensitivity analysis of the double eigenvalues, whose number is finite and relatively small. Owing to the simplicity of the matrices of the unperturbed system the conditions describing eigenvalue assignment have a simple and explicit form and are expressed through the elements of the stiffness matrix. In the subsequent sections we show that in the presence of the damping and non-conservative positional forces similar conclusions can be made not only with respect to the Campbell diagram but also to the decay rate plots.

\section{Stiffness and damping modifications}

Assuming $\nu=0$ in the equation (21), then separating real and imaginary parts in it and equating the real part of the perturbed eigenvalues to zero we find the linear approximation to the surface separating stability and instability domains of the axisymmetric rotor perturbed by the potential and damping forces coming from its interaction with the stator

$$
\begin{aligned}
{\left[\operatorname{tr} \mathbf{D}_{s s}\right.} & \left.\operatorname{tr} \mathbf{D}_{t t}\left[4(s \varepsilon-t \sigma) \Delta \Omega+\kappa\left(\frac{\operatorname{tr} \mathbf{K}_{s s}}{\alpha \omega_{s}}-\frac{\operatorname{tr} \mathbf{K}_{t t}}{\beta \omega_{t}}\right)\right]-\kappa Q\left(\frac{\operatorname{tr} \mathbf{D}_{s s}}{\alpha \omega_{s}}-\frac{\operatorname{tr} \mathbf{D}_{t t}}{\beta \omega_{t}}\right)\right]^{2} \\
& +\kappa^{2}\left(\frac{\operatorname{tr} \mathbf{D}_{s s}}{\alpha \omega_{s}}+\frac{\operatorname{tr} \mathbf{D}_{t t}}{\beta \omega_{t}}\right)^{2}\left[\operatorname{tr} \mathbf{D}_{s s} \operatorname{tr} \mathbf{D}_{t t}\left(\left(\operatorname{tr} \mathbf{K}_{s t} \mathbf{I}_{\varepsilon \sigma}\right)^{2}+\left(\operatorname{tr} \mathbf{K}_{s t} \mathbf{J}_{\varepsilon \sigma}\right)^{2}\right)-Q^{2}\right] \\
= & \delta^{2} \omega_{0}^{2}\left(\frac{\operatorname{tr} \mathbf{D}_{s s}}{\alpha \omega_{s}}+\frac{\operatorname{tr} \mathbf{D}_{t t}}{\beta \omega_{t}}\right)^{2} \operatorname{tr} \mathbf{D}_{s s} \operatorname{tr} \mathbf{D}_{t t}\left(\left(\operatorname{tr} \mathbf{D}_{s t} \mathbf{I}_{\varepsilon \sigma}\right)^{2}\right. \\
& \left.+\left(\operatorname{tr} \mathbf{D}_{s t} \mathbf{J}_{\varepsilon \sigma}\right)^{2}-\operatorname{tr} \mathbf{D}_{s s} \operatorname{tr} \mathbf{D}_{t t}\right),
\end{aligned}
$$

where $Q=\operatorname{tr} \mathbf{K}_{s t} \mathbf{I}_{\varepsilon \sigma} \operatorname{tr} \mathbf{D}_{s t} \mathbf{I}_{\varepsilon \sigma}+\operatorname{tr} \mathbf{K}_{s t} \mathbf{J}_{\varepsilon \sigma} \operatorname{tr} \mathbf{D}_{s t} \mathbf{J}_{\varepsilon \sigma}$. In general, equation (34) describes a conical surface in the $(\Omega, \kappa, \delta)$-space. Orientation of the cone depends on the structure of the matrices $\mathbf{K}$ and $\mathbf{D}$, that is, on the modification of the stiffness and damping characteristics of the stator.

For the crossings of the Campbell diagram situated on the axis $\Omega=0$ the expression (34) greatly simplifies. Indeed, substituting $s=t, \sigma=-\varepsilon$, and $\alpha=\beta$ into equation (34) and rearranging its terms we find

$$
\kappa^{2} A_{s s}+s^{2} \Omega^{2}\left(2 \omega_{s} \operatorname{tr} \mathbf{D}_{s s}\right)^{2}=-\operatorname{det} \mathbf{D}_{s s}\left(\omega_{s} \operatorname{tr} \mathbf{D}_{s s}\right)^{2} \delta^{2},
$$


with the coefficient $A_{s s}$ defined as

$$
\begin{aligned}
A_{s s}= & \operatorname{det} \mathbf{D}_{s s}\left(\rho_{1}\left(\mathbf{K}_{s s}\right)-\rho_{2}\left(\mathbf{K}_{s s}\right)\right)^{2} \\
& +\left(d_{2 s-1,2 s}\left(k_{2 s-1,2 s-1}-k_{2 s, 2 s}\right)-k_{2 s-1,2 s}\left(d_{2 s-1,2 s-1}-d_{2 s, 2 s}\right)\right)^{2},
\end{aligned}
$$

where $\rho_{1}\left(\mathbf{K}_{s s}\right)$ and $\rho_{2}\left(\mathbf{K}_{s s}\right)$ are the eigenvalues of the $2 \times 2$ submatrix $\mathbf{K}_{s s}$ of the $4 \times 4$ stiffness matrix $\mathbf{K}$.

For the definite submatrix $\mathbf{D}_{s s}$ we have $A_{s s}>0$ because $\operatorname{det} \mathbf{D}_{s s}>0$. Consequently, for $\delta \mathbf{D}_{s s}>0$ the stability conditions

$$
\delta \operatorname{tr} \mathbf{D}_{s s}>0, \quad \kappa^{2} A_{s s}+s^{2} \Omega^{2}\left(2 \omega_{s} \operatorname{tr} \mathbf{D}_{s s}\right)^{2}>-\operatorname{det} \mathbf{D}_{s s}\left(\omega_{s} \operatorname{tr} \mathbf{D}_{s s}\right)^{2} \delta^{2}
$$

are always fulfilled. Since $\operatorname{det} \mathbf{D}_{s s}<0$ in case of indefinite matrix $\mathbf{D}_{s s}$, the inequalities (37) indicate that the flutter instability domain for $A_{s s}>0$ is inside the conical surface extended along the $\delta$-axis and the stability domain is adjacent to the cone's skirt selected by the condition $\delta \operatorname{tr} \mathbf{D}_{s s}>0$, see Figure 2(a).

Figure 2 Domains of the subcritical flutter instability associated with the doublet $i \omega_{s}$ situated on the axis $\Omega=0$ of the Campbell diagram for the indefinite submatrix $\mathbf{D}_{s s}$ in the absence of the non-conservative positional forces when: (a) $A_{s s}>0$; (b) $A_{s s}=0$ and (c) $A_{s s}<0$
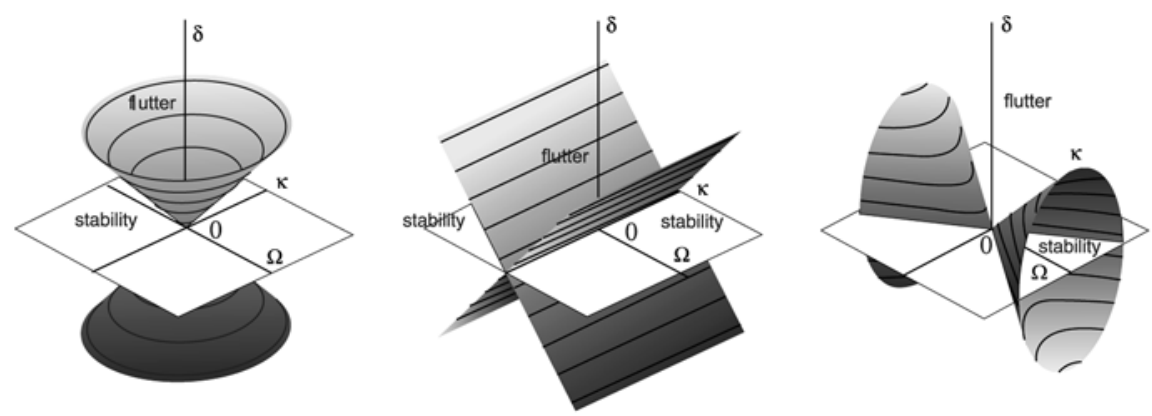

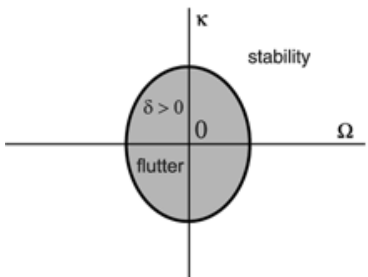

(a)

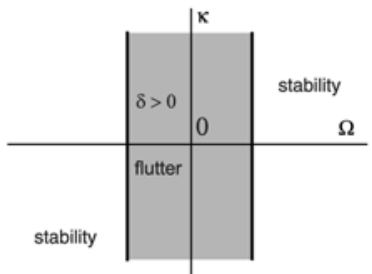

(b)

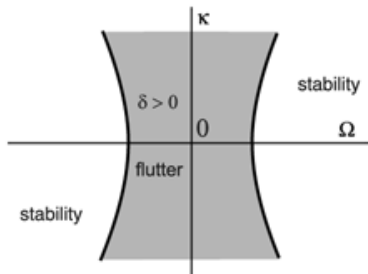

(c)

The conical domain is stretched along the $\kappa$-axis when $A_{s s}$ tends to zero and it is transformed into a dihedral angle when $A_{s s}=0$, as shown in Figure 2(b). With the further decrease in $A_{s s}$ the dihedral angle is again wrapped into the conical surface which is then extended along the $\Omega$-axis, Figure 2(c). The domain of asymptotic stability is inside the half of the cone selected by the inequality $\delta \operatorname{tr} \mathbf{D}_{s s}>0$. Note that the threshold $A_{s s}=0$, separating the indefinite damping matrices, coincides with that found first by Kirillov (2007a) from the criteria of Routh and Hurwitz for a general two-dimensional non-conservative gyroscopic system with dissipation. 
In the plane $(\Omega, \kappa)$ for a fixed $\delta>0$ the instability domain has, respectively, the form of an ellipse, Figure 2(a), a stripe, Figure 2(b), or a region contained between the branches of a hyperbola, Figure 2(c). The latter picture shows that even considerable stiffness modification separating the eigenvalue branches of the undamped system, cannot always prevent from the development of the flutter instability in the presence of indefinite damping, which originates from the brake pads with the negative friction-velocity gradient (Hagedorn, 1988; Spurr, 1961).

Bifurcation of the stability diagrams with the change of the entries of the matrices $\mathbf{D}$ and $\mathbf{K}$ can also explain the well-known unsatisfactory reproducibility of experiments with disk brake. Indeed, some parameters like rotational speed and pressure on the brake pads can be regulated precisely, while the topography of the pads' surface as well as the material properties of the pads undergo uncontrollable changes from one run of the experiment to the other owing to the heating, cooling, and wear. As a consequence, the very same values of stiffness parameter $\kappa$ and rotational speed $\Omega$, which yield flutter instability and squeal for $A_{s s}<0$, Figure 2(c), make the brake quiet for $A_{s s}>0$ owing to the qualitative change of the stability diagram with the change in the structure of the matrices $\mathbf{D}$ and $\mathbf{K}$, Figure 2(a). This effect becomes even more pronounced in the presence of non-conservative positional forces, because, as we show in the next section, the conical stability boundaries are not structurally stable under small perturbations $\nu \mathbf{N}$.

Now we demonstrate how the developed perturbation technique allows one to predict the onset of the flutter instability and the critical value of the eigenfrequency at the onset. The real and imaginary parts of the eigenvalues $\lambda$ that are born after the splitting of the doublet $i \omega_{s}$ situated on the axis $\Omega=0$ are

$$
\operatorname{Re} \lambda=-\frac{\operatorname{tr} \mathbf{D}_{s s}}{4} \delta \pm \sqrt{\frac{|c|+\operatorname{Re} c}{2}}, \quad \operatorname{Im} \lambda=\omega_{s}+\frac{\operatorname{tr} \mathbf{K}_{s s}}{4 \omega_{s}} \kappa \pm \sqrt{\frac{|c|-\operatorname{Re} c}{2}},
$$

with

$$
\begin{aligned}
\operatorname{Re} c & =\left(\frac{\mu_{1}\left(\mathbf{D}_{s s}\right)-\mu_{2}\left(\mathbf{D}_{s s}\right)}{4}\right)^{2} \delta^{2}-\left(\frac{\rho_{1}\left(\mathbf{K}_{s s}\right)-\rho_{2}\left(\mathbf{K}_{s s}\right)}{4 \omega_{s}}\right)^{2} \kappa^{2}-\Omega^{2} s^{2}, \\
\operatorname{Im} c & =\delta \kappa \frac{2 \operatorname{tr} \mathbf{K}_{s s} \mathbf{D}_{s s}-\operatorname{tr} \mathbf{K}_{s s} \operatorname{tr} \mathbf{D}_{s s}}{8 \omega_{s}},
\end{aligned}
$$

where $\mu_{1}\left(\mathbf{D}_{s s}\right)$ and $\mu_{2}\left(\mathbf{D}_{s s}\right)$ are the eigenvalues of the submatrix $\mathbf{D}_{s s}$.

With the variation of the speed $\Omega$ the eigenvalues (38) move along the branches of the hyperbola in the complex plane

$$
\left(\operatorname{Re} \lambda+\frac{\operatorname{tr} \mathbf{D}_{s s}}{4} \delta\right)\left(\operatorname{Im} \lambda-\omega_{s}-\frac{\operatorname{tr} \mathbf{K}_{s s}}{4 \omega_{s}} \kappa\right)=-\frac{\operatorname{Im} c}{2} .
$$

When $\operatorname{Im} c=0$ the strong modal resonance occurs (Dobson et al., 2001) with the coupling of eigenvalues at $\operatorname{Re} \lambda=-\frac{\operatorname{tr} \mathbf{D}_{s s}}{4} \delta$ and $\operatorname{Im} \lambda=\omega_{s}+\frac{\operatorname{tr} \mathbf{K}_{s s}}{4 \omega_{s}} \kappa$. This new double eigenvalue has only one eigenvector and strongly influences the eigenvalue trajectories for small values of $\kappa$ and $\delta$ when $\operatorname{Im} c \neq 0$ and is known to be a precursor to flutter instability in hydrodynamics (Jones, 1988) and in electrical circuits (Dobson et al., 2001). The branch, which crosses the imaginary axis from left to right, is determined 
by the sign of $\operatorname{Im} c$. Assuming $\operatorname{Re} \lambda=0$ in equation (40), we find the eigenfrequency of the mode at the onset of self-excited vibrations

$$
\omega_{c r}=\omega_{s}+\kappa \frac{\operatorname{tr} \mathbf{K}_{s s} \operatorname{tr} \mathbf{D}_{s s}-\operatorname{tr} \mathbf{K}_{s s} \mathbf{D}_{s s}}{2 \omega_{s} \operatorname{tr} \mathbf{D}_{s s}} .
$$

The corresponding value of the rotational speed $\Omega$ is found from the equation (35).

As we have shown in the previous section the stiffness variation with the matrix $\mathbf{K}=\mathbf{K}_{3}$ leads to the divergence instability near the crossing $(5,0)$, see Figures 1(d), (f) and 3(a), and to the veering of eigenvalue branches in the Campbell diagram near the crossing $(0,5)$ as shown in Figure 1(d).

Figure $3 \mathbf{D}=\mathbf{D}_{1}, \mathbf{K}=\mathbf{K}_{3}, \nu=0$ : Numerically calculated eigenvalue branches (black circles) and their approximations (37) and (39) (red lines) for (a) $\kappa=0.8$ and $\delta=0$; (b) $\kappa=0 ; \delta=0.2$ and (c)-(f) $\kappa=0.8$ and $\delta=0.2$ (see online version for colours)

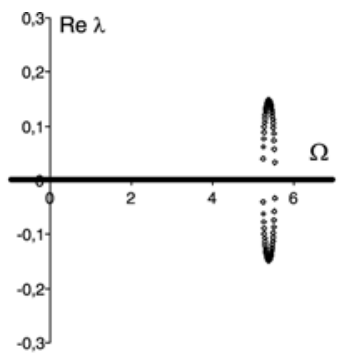

(a)

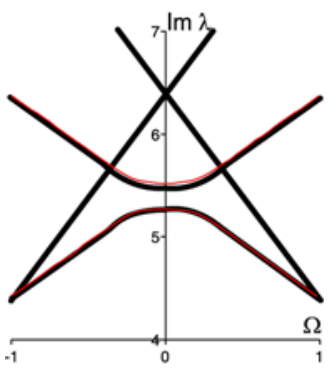

(d)

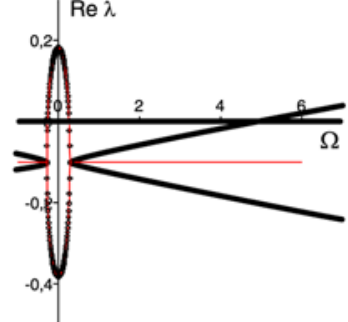

(b)

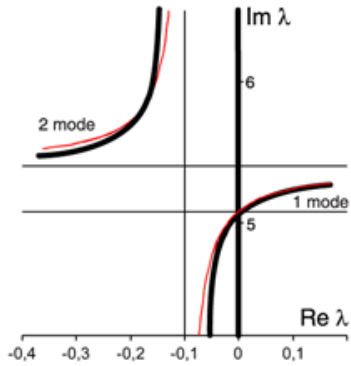

(e)

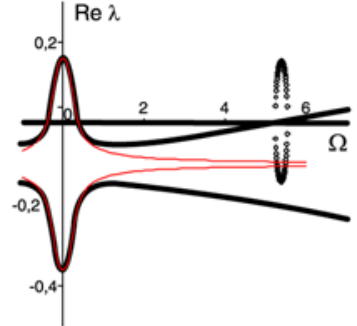

(c)

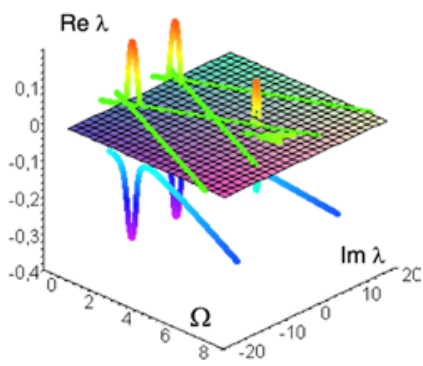

(f)

Let us consider the two damping matrices

$$
\mathbf{D}_{1}=\left(\begin{array}{cccc}
-1 & 2 & 0 & 0 \\
2 & 3 & 0 & 0 \\
0 & 0 & 0 & 0 \\
0 & 0 & 0 & 0
\end{array}\right), \quad \mathbf{D}_{2}=\left(\begin{array}{cccc}
-1 & 0.5 & 0 & 3 \\
0.5 & 3 & 2 & 1 \\
0 & 2 & -3 & 2 \\
3 & 1 & 2 & 4
\end{array}\right)
$$

In the absence of the stiffness perturbation $(\kappa=0)$, the damping modification with the matrix $\mathbf{D}=\mathbf{D}_{1}$ yields a non-trivial decay rate plot with the bubble of instability in the vicinity of $\Omega=0$, Figure 3(b). The magnitude of the positive real part for the unstable arc of the bubble is of the same order as for the unstable eigenvalues caused by the stiffness variation with the matrix $\mathbf{K}=\mathbf{K}_{3}$ near the crossing $(5,0)$, Figure 3(a). Consequently, the indefinite damping submatrix $\mathbf{D}_{s s}$ is the source of flutter 
instability as dangerous in the subcritical speed range as the stiffness variation is in the supercritical speed range, in agreement with the results of Kirillov (2008b) obtained for the systems with two degrees of freedom.

Moreover, numerical computations show that even if the eigenvalue branches in the subcritical speed range of the Campbell diagram are well-separated by the stiffness modification $\mathbf{P}+\kappa \mathbf{K}_{3}$, the inclusion of dissipation with the indefinite matrix $\mathbf{D}=\mathbf{D}_{1}$ causes flutter instability in the vicinity of the crossing $(0,5)$, see Figure 3(c)-(f). The stiffness variation leads to the partial destruction of the bubble of instability created by the indefinite damping, Figure 3(c). The arc of unstable eigenvalues, corresponding to the first vibration mode, however, persists. Comparison of the numerical results with the predictions given by the analytical formulas (38)-(40) shows a very good qualitative and quantitative agreement visible in Figure 3.

For the matrices $\mathbf{K}=\mathbf{K}_{3}$ and $\mathbf{D}=\mathbf{D}_{1}$ we find from the equation (36) that $A_{11}=-48$ and hence the conical stability boundary

$$
48 \kappa^{2}+700 \delta^{2}=400 \Omega^{2},
$$

calculated with the use of the expression (35), is oriented along the $\Omega$-axis as shown in Figure 2(c). Such a geometry of the stability domain yields flutter instability in the vicinity of the crossing $(0,5)$ for nonzero values of the parameters $\delta$ and $\kappa$, which is confirmed by the numerical calculations shown in Figure 3.

In the case when $s=t, \sigma=-\varepsilon$, and $\alpha=-\beta$, corresponding to the doublets situated on the $\Omega$-axis, the conical instability domain degenerates into the half-space

$$
\kappa<\frac{2 \varepsilon s \omega_{s} \operatorname{tr} \mathbf{D}_{s s}}{2 k_{2 s-1,2 s} d_{2 s-1,2 s}-k_{2 s-1,2 s-1} d_{2 s, 2 s}-k_{2 s, 2 s} d_{2 s-1,2 s-1}} \Delta \Omega .
$$

In the vicinity of the crossing $(5,0)$ the formula (44) predicts instability for $\Omega>5+0.1 \kappa$ due to perturbation by the matrices $\mathbf{K}_{3}$ and $\mathbf{D}_{1}$. This agrees with the results of the numerical calculations shown in Figure 3(c).

Now we modify the damping matrix so that $\mathbf{D}=\mathbf{D}_{2}$. Comparing to the matrix $\mathbf{D}_{1}$ the entry $d_{12}$ is changed from $d_{12}=2$ in $\mathbf{D}_{1}$ to $d_{12}=0.5$ in $\mathbf{D}_{2}$. In both cases the submatrix $\mathbf{D}_{11}$ is indefinite but the coefficient $A_{11}=12$ in the latter case contrary to $A_{11}=-48$ in the former. This yields the conical flutter domain oriented along the $\delta$-axis with the boundary

$$
12 \kappa^{2}+400 \Omega^{2}=325 \delta^{2},
$$

as shown in Figures 2(a) and 4(a). This example clearly demonstrates how the relatively small variation of the coefficient $d_{12}$ qualitatively changes the instability domain from one with the boundary described by the equation (43) to that with the boundary described by the equation (45). Therefore, the system which was unstable for $\delta=0.1$ and $\kappa=0.7$ when $\mathbf{D}=\mathbf{D}_{1}$ becomes stable for the same values of these parameters when $\mathbf{D}=\mathbf{D}_{2}$. The corresponding eigenvalue branches of the first and second modes have now negative real parts, which is confirmed also numerically in Figure 4(c) and (d).

The non-trivial submatrices $\mathbf{D}_{12}$ and $\mathbf{D}_{22}$ of the matrix $\mathbf{D}_{2}$ cause the excitation of the unstable modes in the vicinity of the crossings $(1 / 3,16 / 3)$ and $(0,6)$, 
see Figure 4(c) and (d). The corresponding flutter instability domains shown in Figure 4(a) are dihedral-angle-like with the boundaries

$$
(1 / 3,16 / 3):(24 \Omega-8)^{2}-\frac{295936}{675} \delta^{2}=0 ; \quad(0,6): \Omega^{2}-\delta^{2}=0 .
$$

For $\delta=0.1$ and $\kappa=0.7$ the matrix $\mathbf{D}_{2}$ selectively inhibits instability of the first two modes in the vicinity of $\Omega=0$. Instead, the fourth mode is unstable near $\Omega=0$ and the third one makes the excursion to the right side of the complex plane near $\Omega=1 / 3$, see Figure 4(c) and (d). Approximation (21) shown in Figure 4 by the red lines simulates well the behaviour of eigenvalues both qualitatively and quantitatively.

Figure $4 \mathbf{D}=\mathbf{D}_{2}, \mathbf{K}=\mathbf{K}_{3}, \nu=0$ : (a) linear approximation to the flutter domains and (b)-(d) numerically calculated eigenvalue branches (black circles) and their approximations (37) and (39) (red lines) for $\kappa=0.7$ and $\delta=0.1$ (see online version for colours)

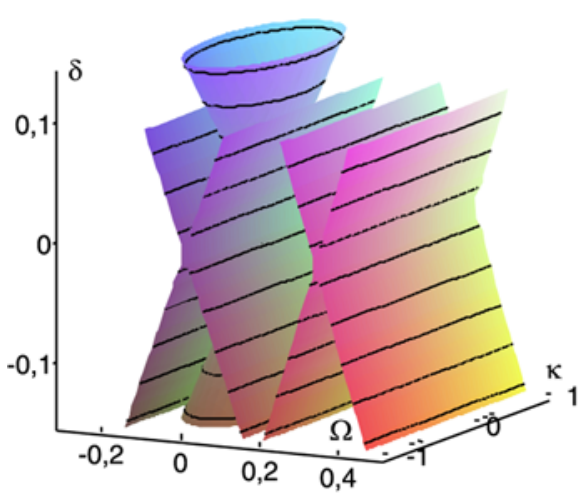

(a)

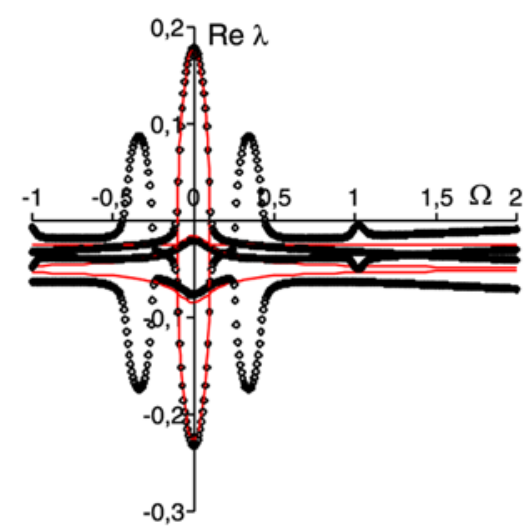

(c)

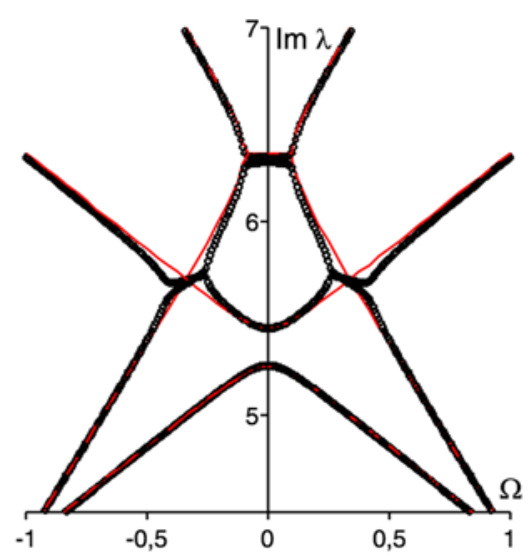

(b)

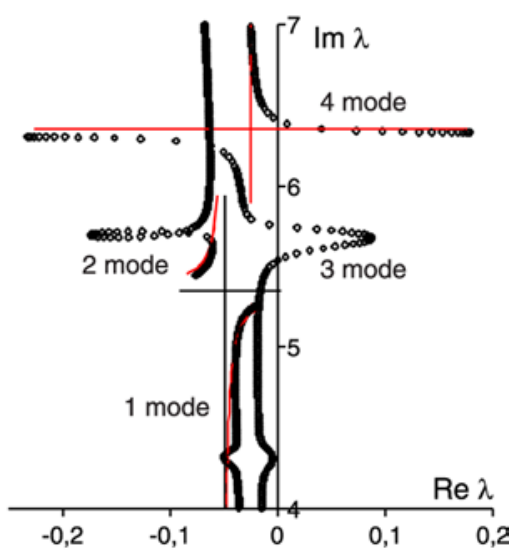

(d)

Therefore, the influence of damping on the real parts of the eigenvalues is not homogeneous with respect to $\Omega$. The smaller the gap between the imaginary parts of 
the eigenvalues in the Campbell diagram the bigger the increment to the real parts due to development of the imperfect bubbles of instability in the decay rate plot, Figure 4(c). Numerous examples of such dissipation-induced imperfect bubbles of instability developed in the supercritical speed range of high-speed propellers and bladed disks of turbines can be found in Genta (2007). Numerical calculations of Yang and Hutton (1995) revealed them in the subcritical range for the rotating circular string passing through the eyelet with friction (see also Kirillov, 2008b). Xiong et al. (2002) found the same effect for the rotating disk in frictional contact with the point-wise stationary load system. Due to the ability of damping to create a strong inhomogeneous increment to the real parts of the eigenvalues, it has to be considered as a serious reason for the self-excited vibrations in the subcritical speed range.

The relation of the pronounced decay rates to the small spectral gaps is explained by the conical singularities of the stability boundaries associated with the doublets in the Campbell diagrams of the unperturbed isotropic rotor. Even for the fixed structure of the perturbing matrices the geometry of the stability frontiers in the $(\Omega, \kappa, \delta)$-space is complicated by the different orientation of the cones leading to the controversial requirements to the choice of the intensities of the perturbations $\Omega, \kappa$, and $\delta$ (cf. Ouyang, 2008). Sensitivity analysis of the doublets and approximation of the stability boundaries plays the role of a 'cartographic plotting' of the obstacles for the stabilising eigenvalue assignment due to stiffness and damping modifications. Possible bifurcations of the stability domains due to modification of the structure of the perturbing matrices can also be predicted by the sensitivity analysis. In the next section we study such obstacles in the most complicated case when the non-conservative positional forces are taken into account.

\section{Modifying the matrices of stiffness, damping, and circulatory forces}

In general, sensitivity of a doublet with respect to the perturbation by the matrices of stiffness, damping, and circulatory forces is described by the formula (21). For brevity we analyse in this section only the crossings situated on the axis $\Omega=0$. Substituting $s=t, \varepsilon \neq \sigma$, and $\alpha=\beta$ into (21) we find that the perturbed eigenvalues are

$$
\operatorname{Re} \lambda=-\frac{\operatorname{tr} \mathbf{D}_{s s}}{4} \delta \pm \sqrt{\frac{|c|+\operatorname{Re} c}{2}}, \quad \operatorname{Im} \lambda=\omega_{s}+\frac{\operatorname{tr} \mathbf{K}_{s s}}{4 \omega_{s}} \kappa \pm \sqrt{\frac{|c|-\operatorname{Re} c}{2}},
$$

with

$$
\begin{aligned}
& \operatorname{Re} c=\left(\frac{\mu_{1}\left(\mathbf{D}_{s s}\right)-\mu_{2}\left(\mathbf{D}_{s s}\right)}{4}\right)^{2} \delta^{2}-\left(\frac{\rho_{1}\left(\mathbf{K}_{s s}\right)-\rho_{2}\left(\mathbf{K}_{s s}\right)}{4 \omega_{s}}\right)^{2} \kappa^{2}-s^{2} \Omega^{2}+\nu^{2} \frac{n_{2 s-1,2 s}^{2}}{4 \omega_{s}^{2}} \\
& \operatorname{Im} c=\Omega \nu \frac{s n_{2 s-1,2 s}}{\omega_{s}}-\delta \kappa \frac{2 \operatorname{tr} \mathbf{K}_{s s} \mathbf{D}_{s s}-\operatorname{tr} \mathbf{K}_{s s} \operatorname{tr} \mathbf{D}_{s s}}{8 \omega_{s}}
\end{aligned}
$$

In the presence of non-conservative positional forces $(\nu \neq 0)$, stability conditions, following from equations (47) and (48) under the requirement $\operatorname{Re} \lambda<0$, are $\delta \operatorname{tr} \mathbf{D}_{s s}>0$ and $B_{s s}>0$, where

$$
\begin{aligned}
B_{s s}= & \left(2 \Omega s\left(\delta^{2} \omega_{s}^{2}\left(\operatorname{tr} \mathbf{D}_{s s}\right)^{2}-4 \nu^{2} n_{2 s-1,2 s}^{2}\right)\right. \\
& \left.+\delta\left(2 \operatorname{tr} \mathbf{K}_{s s} \mathbf{D}_{s s}-\operatorname{tr} \mathbf{K}_{s s} \operatorname{tr} \mathbf{D}_{s s}\right) \kappa \nu n_{2 s-1,2 s}\right)^{2}
\end{aligned}
$$




$$
\begin{aligned}
& +\delta^{2}\left(\operatorname{tr} \mathbf{D}_{s s}\right)^{2}\left(A_{s s} \delta^{2} \omega_{s}^{2}-\nu^{2} n_{2 s-1,2 s}^{2}\left(\rho_{1}\left(\mathbf{K}_{s s}\right)-\rho_{2}\left(\mathbf{K}_{s s}\right)\right)^{2}\right) \kappa^{2} \\
& -\delta^{2}\left(\operatorname{tr} \mathbf{D}_{s s}\right)^{2}\left(\delta^{2} \omega_{s}^{2}\left(\operatorname{tr} \mathbf{D}_{s s}\right)^{2}-4 \nu^{2} n_{2 s-1,2 s}^{2}\right)\left(\nu^{2} n_{2 s-1,2 s}^{2}-\delta^{2} \omega_{s}^{2} \operatorname{det} \mathbf{D}_{s s}\right) .
\end{aligned}
$$

The coefficient $A_{s s}$ defined in equation (36) we represent in the form

$$
A_{s s}=\frac{\left(\left(\operatorname{tr} \mathbf{D}_{s s}\right)^{2}-16 \beta_{\circ}^{2}\right)\left(\rho_{1}\left(\mathbf{K}_{s s}\right)-\rho_{2}\left(\mathbf{K}_{s s}\right)\right)^{2}}{4}
$$

with

$$
\beta_{\circ}=\frac{2 \operatorname{tr} \mathbf{K}_{s s} \mathbf{D}_{s s}-\operatorname{tr} \mathbf{K}_{s s} \operatorname{tr} \mathbf{D}_{s s}}{4\left(\rho_{1}\left(\mathbf{K}_{s s}\right)-\rho_{2}\left(\mathbf{K}_{s s}\right)\right)} .
$$

In the assumption that $\nu=0$ the inequality $B_{s s}>0$ is reduced to the second one of the conditions (37).

For the fixed parameters $\delta$ and $\nu$ the equation $B_{s s}=0$ generically describes either an ellipse or a hyperbola in the $(\Omega, \kappa)$-plane, Figure 5(b) and (d). For $A_{s s}>0$ and sufficiently big $\delta$, the domain of subcritical flutter is inside the ellipse $B_{s s}=0$, and the domain of asymptotic stability is outside the ellipse similarly to the system without non-conservative positional forces, whose stability diagram is shown in Figure 2(a). With the decrease in $\delta$ for the fixed $\nu$ the ellipse is rotated around the origin in the $(\Omega, \kappa)$-plane and simultaneously it is stretched along one of its main axes. At the threshold $A_{s s} \delta^{2} \omega_{s}^{2}=\nu^{2} n_{2 s-1,2 s}^{2}\left(\rho_{1}\left(\mathbf{K}_{s s}\right)-\rho_{2}\left(\mathbf{K}_{s s}\right)\right)^{2}$ the ellipse is transformed into two parallel lines, which with the further decrease in $\delta$ are bent into two branches of hyperbola $B_{s s}=0$. Then, the flutter instability domain lies between the two branches, Figure 5(b). When $\delta$ tends to zero, the hyperbolic branches are shrunk to the union of the intervals $\left(-\infty,-\kappa_{\circ}\right] \bigcup\left[\kappa_{\circ}, \infty\right)$ of the $\kappa$-axis, where the critical value

$$
\kappa_{\circ}=\frac{2 \nu n_{2 s-1,2 s}}{\rho_{1}\left(\mathbf{K}_{s s}\right)-\rho_{2}\left(\mathbf{K}_{s s}\right)}
$$

follows from the conditions $\operatorname{Re} c=0$ and $\operatorname{Im} c=0$ implying existence of the double eigenvalues at the points $\left(\Omega=0, \kappa= \pm \kappa_{0}, \delta=0\right)$, which are shown as open circles in Figure 5.

Remarkably, the value (51) of $\kappa_{\circ}$ obtained from the perturbation formulas (47) and (48) coincides with the exact one following from the characteristic equation of the system (6) in the assumption that $\delta=0$ and $\Omega=0$ and that the anti-diagonal subblocks of the perturbing matrices vanish

$$
\lambda^{4}+\left(2 \omega_{s}^{2}+\kappa \operatorname{tr} \mathbf{K}_{s s}\right) \lambda^{2}+\kappa^{2} \operatorname{det} \mathbf{K}_{s s}+\kappa \omega_{s}^{2} \operatorname{tr} \mathbf{K}_{s s}+\nu^{2} n_{2 s-1,2 s}^{2}+\omega_{s}^{4}=0 .
$$

Substituting $\kappa=\kappa_{\circ}$ into (52) yields the frequency of the corresponding double eigenvalues $\pm i \omega_{\circ}$

$$
\omega_{\circ}=\sqrt{\omega_{s}^{2}+\nu n_{2 s-1,2 s} \frac{\rho_{1}\left(\mathbf{K}_{s s}\right)+\rho_{2}\left(\mathbf{K}_{s s}\right)}{\rho_{1}\left(\mathbf{K}_{s s}\right)-\rho_{2}\left(\mathbf{K}_{s s}\right)}} .
$$


Figure 5 Unfolding the conical boundary of subcritical flutter (dashed lines) by the perturbation $\nu \mathbf{N}$ into a couple of Whitney's umbrellas with their level curves depicted for $\delta>0$ when (a)-(b) $A_{s s}>0$ and (c)-(d) $A_{s s}<0$

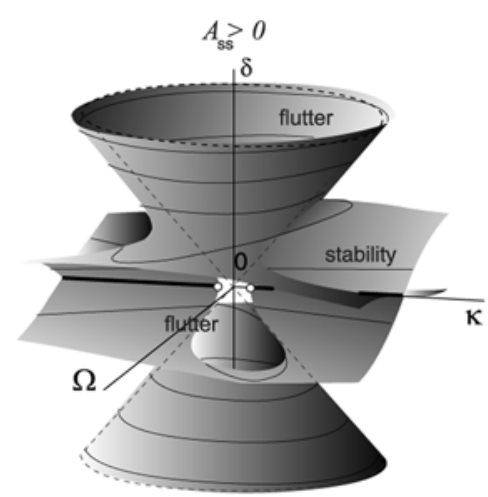

(a)

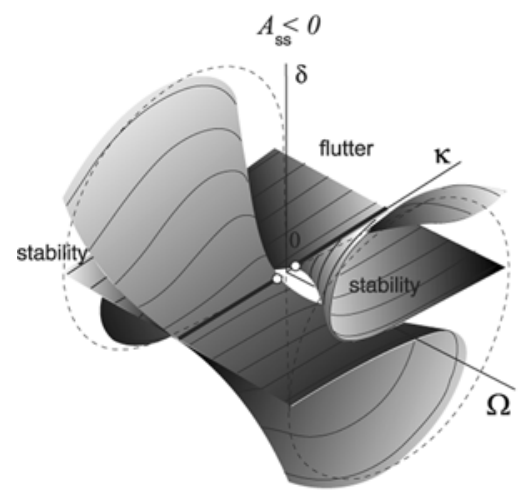

(c)

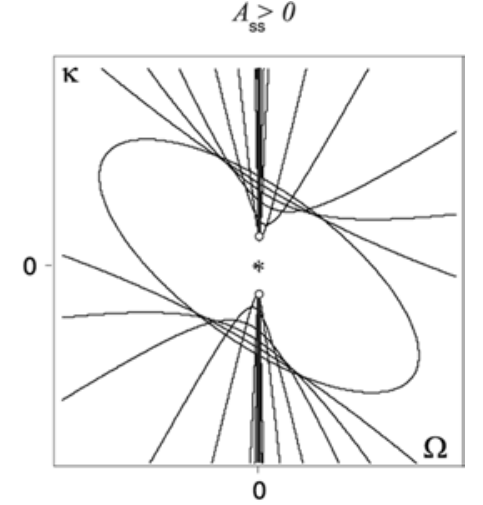

(b)

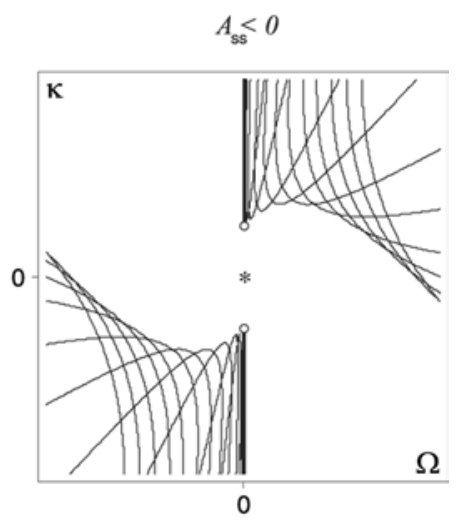

(d)

The double eigenvalue $i \omega_{\circ}$ has the eigenvector $\mathbf{u}_{\circ}$ and the associated vector $\mathbf{u}_{1}$ of the Jordan chain

$$
\mathbf{u}_{\circ}=\left(\begin{array}{c}
k_{2 s-1,2 s-1}-k_{2 s, 2 s} \\
2 k_{2 s-1,2 s}+\rho_{1}\left(\mathbf{K}_{s s}\right)-\rho_{2}\left(\mathbf{K}_{s s}\right)
\end{array}\right), \quad \mathbf{u}_{1}=\frac{2 i \omega_{\circ}\left(\rho_{2}\left(\mathbf{K}_{s s}\right)-\rho_{1}\left(\mathbf{K}_{s s}\right)\right)}{\nu n_{2 s-1,2 s}}\left(\begin{array}{l}
1 \\
0
\end{array}\right),
$$

which are the solutions to the equations

$$
\left(-\omega_{\circ}^{2} \mathbf{I}_{s s}+\mathbf{P}_{s s}+\kappa_{\circ} \mathbf{K}_{s s}\right) \mathbf{u}_{\circ}=0, \quad\left(-\omega_{\circ}^{2} \mathbf{I}_{s s}+\mathbf{P}_{s s}+\kappa_{\circ} \mathbf{K}_{s s}\right) \mathbf{u}_{1}=2 i \omega_{\circ} \mathbf{u}_{\circ} .
$$

Therefore, in the parameter space the coordinates $\left(0, \pm \kappa_{\circ}, 0\right)$ correspond to exceptional points (EPs, Kirillov et al., 2005), at which there exist double eigenvalues with the Jordan chain, Figure 5.

In the vicinity of the EPs the expression $B_{s s}=0$ for the stability boundary yields

$$
\Omega=\frac{4 \beta_{\circ} \kappa \pm \operatorname{tr} \mathbf{D}_{s s} \sqrt{\kappa^{2}-\kappa_{\circ}^{2}}}{4 \kappa_{\circ}} \delta+o(\delta) .
$$


Thus, in the $(\Omega, \delta)$-plane the domain of asymptotic stability is bounded in the first-order approximation by the two straight lines (56). When $\kappa$ goes to $\pm \kappa_{0}$, the slopes of the both lines $\beta:=\Omega / \delta$ tend to the value $\beta= \pm \beta_{\circ}$, where $\beta_{\circ}$ is defined in (50). Extracting $\kappa$ from the equation (56) we find another representation for the stability boundary near exceptional points

$$
\kappa=\kappa_{\circ} \frac{4 \beta \beta_{\circ} \pm \operatorname{tr} \mathbf{D}_{s s} \sqrt{\beta^{2}-\beta_{\circ}^{2}+\left(\frac{\operatorname{tr} \mathbf{D}_{s s}}{4}\right)^{2}}}{4 \beta_{\circ}^{2}-\left(\frac{\operatorname{tr} \mathbf{D}_{s s}}{2}\right)^{2}} \simeq \pm \kappa_{\circ}\left[1+8\left(\frac{\beta \mp \beta_{\circ}}{\operatorname{tr} \mathbf{D}_{s s}}\right)^{2}\right],
$$

which has a canonical for the Whitney's umbrella singularity form $Z=X^{2} / Y^{2}$ (Bottema, 1956; Kirillov, 2007a, 2007b).

Therefore, we explicitly demonstrated that the conical boundary of the domain of subcritical flutter for $A_{s s}>0$ is structurally unstable under the perturbation $\nu \mathbf{N}$. With the increase of $\nu$ the cone opens up and simultaneously the plane $\delta=0$ foliates into two sheets intersecting along the branch cuts $\left( \pm \infty, \pm \kappa_{\circ}\right]$ on the $\kappa$-axis, which are shown as thick lines in Figure 5. The new surface has a couple of Whitney's umbrella singularities at the exceptional points $\left(0, \pm \kappa_{0}, 0\right)$. The domain of asymptotic stability, which for $\nu=0$ was adjacent to the conical domain of subcritical flutter is now wrapped into the pockets of the two Whitney's umbrellas, selected by the inequality $\delta \operatorname{tr} D>0$. With the increase in $\delta$ the stability boundary gradually tends to the conical surface with the flutter instability inside it, Figure 5(a).

Inclusion of the non-conservative forces qualitatively changes the stability diagram in the $(\Omega, \kappa)$-plane transforming the elliptic flutter domain of Figure 2(a) to a larger one located between the hyperbolic branches, Figure 5(b). For $\nu=0$ and $\delta=0$ the $(\Omega, \kappa)$-plane is stable, while for $\nu \neq 0$ and $\delta \rightarrow 0$ the stability domain dramatically shrinks to the branch cuts $\left( \pm \infty, \pm \kappa_{\circ}\right]$. Consequently, under small perturbation $\nu \mathbf{N}$ a point in the $(\Omega, \kappa)$-plane, which was in the stability domain for $\nu=0$ can suddenly find itself in the instability region when $\nu \neq 0$, similarly to the scenario described in the previous section.

For $A_{s s}<0$ the conical stability boundary of Figure 2(c) unfolds into two surfaces with the Whitney's umbrella singularities at the exceptional points $\left(0, \pm \kappa_{\circ}, 0\right)$ as shown in Figure 5(c). The local approximations to the surfaces near the singularities are given by the same equation (56), where $\beta_{\circ}$ has a value different from the case when $A_{s s}>0$. For $\nu=0$ and $\delta \neq 0$ the stability domain in the $(\Omega, \kappa)$-plane is inside of the two hyperbolic regions extended along the $\Omega$-axis, as shown in Figure 2(c). When $\nu \neq 0$ with the decrease of $\delta$ the stability domain rotates around the origin until it is completely reoriented and shrunk into the branch cuts $\left( \pm \infty, \pm \kappa_{\circ}\right]$ extended along the $\kappa$-axis, Figure 5(d). Due to such a reorientation one can again observe sudden stabilisation/destabilisation at the very same values of $\Omega$ and $\kappa$ when $\nu$ is slightly changed.

Finally, we demonstrate how the obtained knowledge on the geometry of the instability domain improves the understanding of the perturbed eigenvalue behaviour. We calculate numerically the eigenvalue branches for the matrices $\mathbf{K}=\mathbf{K}_{3}, \mathbf{D}=\mathbf{D}_{1}$ and $\mathbf{N}=\mathbf{N}_{1}$, where

$$
\mathbf{N}_{1}=\left(\begin{array}{cccc}
0 & -1 & 0 & 0 \\
1 & 0 & 0 & 0 \\
0 & 0 & 0 & 0 \\
0 & 0 & 0 & 0
\end{array}\right)
$$


In this case the crossing $(0,5)$ of the Campbell diagram is affected by the perturbation. As in the previous section the constant $A_{11}=-48$ so that in the absence of the non-conservative positional forces the stability boundary is a cone (43) oriented along the $\Omega$-axis. This leads to the typical instability arc visible in Figure 6(a).

In the absence of damping $\delta=0$ the gyroscopic system with the potential and non-conservative positional forces cannot be asymptotically stable in accordance with the theorem by Lakhadanov (1975). It is unstable almost everywhere in the space of the parameters and can be only marginally stable on the set of measure zero in the parameter space. This result is illustrated in Figure 6(b) and (d). In Figure 6(b) the real parts cross at the origin in the $(\Omega, \operatorname{Re} \lambda)$-plane, because the value of $\kappa=0.9$ exceeds $\kappa_{\circ}=0.1$ so that with the variation of $\Omega$ at $\kappa=0.9$ we intersect the branch cut $\left[\kappa_{\circ}, \infty\right)$ in the $(\Omega, \kappa)$-plane. For $\delta=0$ the system is marginally stable at the points of the branch cut, which is the set of measure zero, and unstable at all other points of the parameter plane. In Figure 6(b) the real parts do not cross in the $(\Omega, \operatorname{Re} \lambda)$-plane, because the value of $\kappa=0.01$ is less than $\kappa_{\circ}=0.1$, so that the branch cut is not intersected with the variation of $\Omega$.

Perturbation formulas for eigenvalues (47) and (48) allow us to plot the real and imaginary parts of the non-conservative gyroscopic system without damping as functions of $\kappa$ and $\Omega$ for a given $\nu \neq 0$, see Figure 7. Hence, the transition from the eigenvalue configuration shown in Figure 6(d) to that shown in Figure 6(b) is just an evolution of the cross-sections of the eigenvalue surfaces of Figure 7 under variation of the parameter $\kappa$. The qualitative changes of the eigenvalue branches due to this evolution is caused by the passage through the exceptional points at $\kappa= \pm \kappa_{\circ}$, where the eigenvalue surfaces have Whitney's umbrella singularities. According to the formulas (47) and (48) the asymptotic behaviour of the real parts of the eigenvalues at $\kappa=\kappa_{\circ}$ is

$$
\operatorname{Re} \lambda= \pm \frac{1}{2} \sqrt{\frac{2 \nu s n_{2 s-1,2 s}}{\omega_{s}} \Omega}+O\left(\Omega^{3 / 2}\right),
$$

in agreement with the results by Yang and Hutton (1995) and Kirillov (2008b) obtained for the rotating circular string passing through the eyelet with friction. For $\kappa<\kappa_{\circ}$ we have constant real parts in agreement with Figure 6(d)

$$
\operatorname{Re} \lambda= \pm \frac{\rho_{1}\left(\mathbf{K}_{s s}\right)-\rho_{2}\left(\mathbf{K}_{s s}\right)}{4 \omega_{s}} \sqrt{\kappa_{\circ}^{2}-\kappa^{2}}+O\left(\Omega^{2}\right),
$$

whereas for $\kappa>\kappa_{\circ}$ the real parts linearly depend on $\Omega$

$$
\operatorname{Re} \lambda= \pm \frac{2 \nu s n_{2 s-1,2 s}}{\left(\rho_{1}\left(\mathbf{K}_{s s}\right)-\rho_{2}\left(\mathbf{K}_{s s}\right)\right) \sqrt{\kappa^{2}-\kappa_{\circ}^{2}}} \Omega+O\left(\Omega^{3}\right)
$$

as shown in Figure 6(b). For $\Omega$ tending to infinity we find that

$$
\operatorname{Re} \lambda= \pm \frac{\nu n_{2 s-1,2 s}}{2 \omega_{s}}+O\left(\Omega^{-2}\right)
$$

which is seen in Figures 6(b), (d) and 7. The singular eigenvalue surface shown in the right picture of Figure 7 is well-known in the physical literature on wave 
164 O.N. Kirillov

Figure $6 \mathbf{K}=\mathbf{K}_{3}, \mathbf{D}=\mathbf{D}_{1}, \mathbf{N}=\mathbf{N}_{1}$ : eigenvalue branches calculated for (a) $\nu=0, \delta=0.02$, and $\kappa=0.9$; (b) $\nu=0.2, \delta=0$, and $\kappa=0.9$; (c) $\nu=0.2, \delta=0.02$, and $\kappa=0.9$;

(d) $\nu=0.2, \delta=0$, and $\kappa=0.01$ and (e) $\nu=0.1, \delta=0.04$, and $\kappa=0$
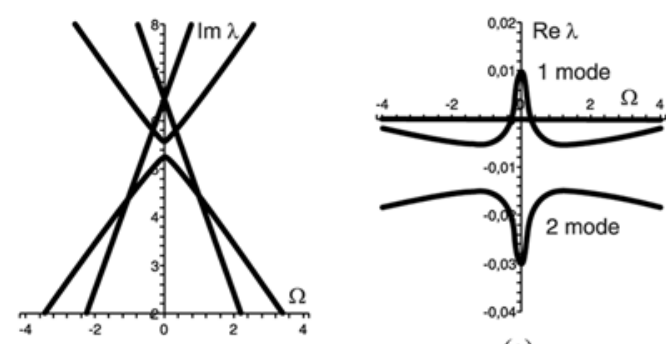

(a)
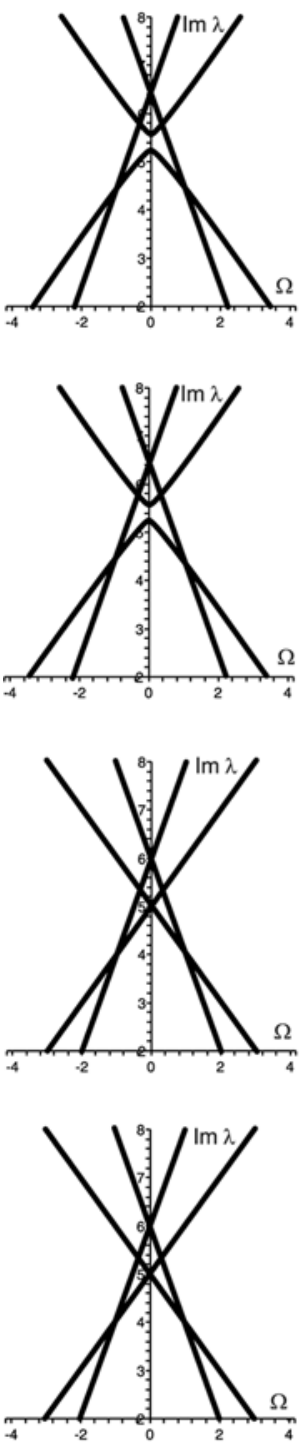

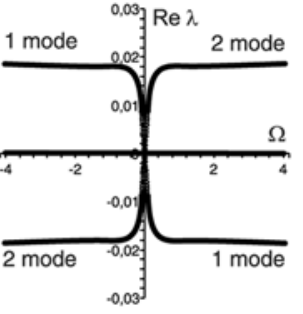

(b)

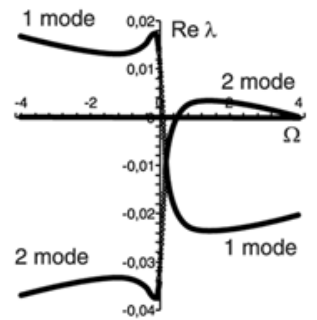

(c)

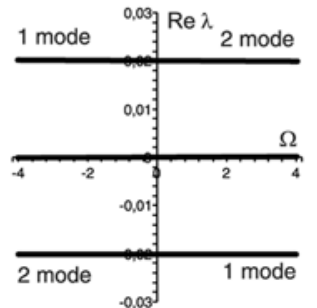

(d)

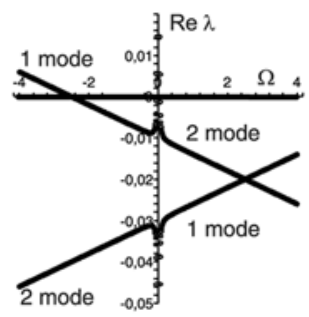

(e)
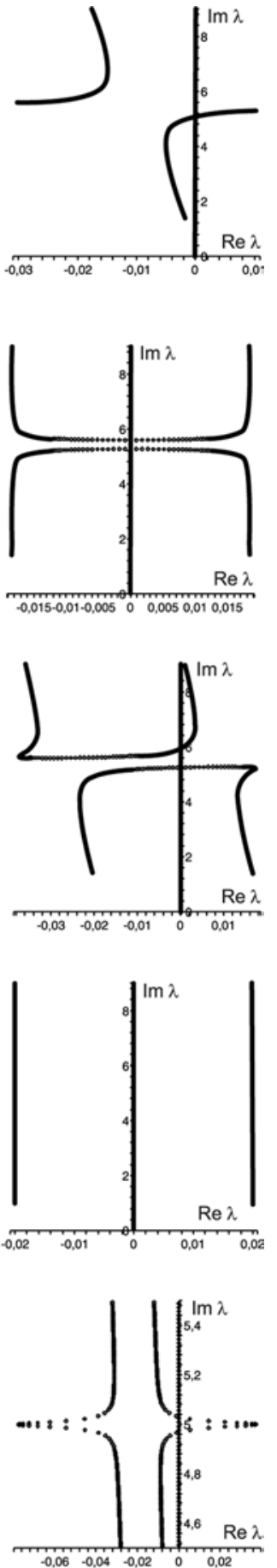
propagation in anisotropic media as the 'double coffee filter' (Kirillov et al., 2005). We notice that eigenvalue surfaces where used in a recent work by Chevillot et al. (2008) who studied the onset of squeal in an aircraft braking system.

Introduction of damping into the gyroscopic system with non-conservative positional forces leads to the origination of the zones of the asymptotic stability, Figure 5(c) and (d). Indeed Figure 6(c) demonstrates that the crossing of the real parts occurs at the negative values thus creating an interval of asymptotic stability on the $\Omega$-axis in agreement with Figure 5(c) and (d). For $\kappa=0$ the instability develops in the vicinity of $\Omega=0$, see Figure 6(e) in full agreement with the qualitative Figure 5(c) and (d) as well as with the analysis of a two-dimensional rotor system by Kirillov (2008b).

Figure 7 Real and imaginary parts of the perturbed eigenvalues as functions of $\kappa$ and $\Omega$ for a given $\nu \neq 0$ in the absence of damping $(\delta=0)$ (see online version for colours)
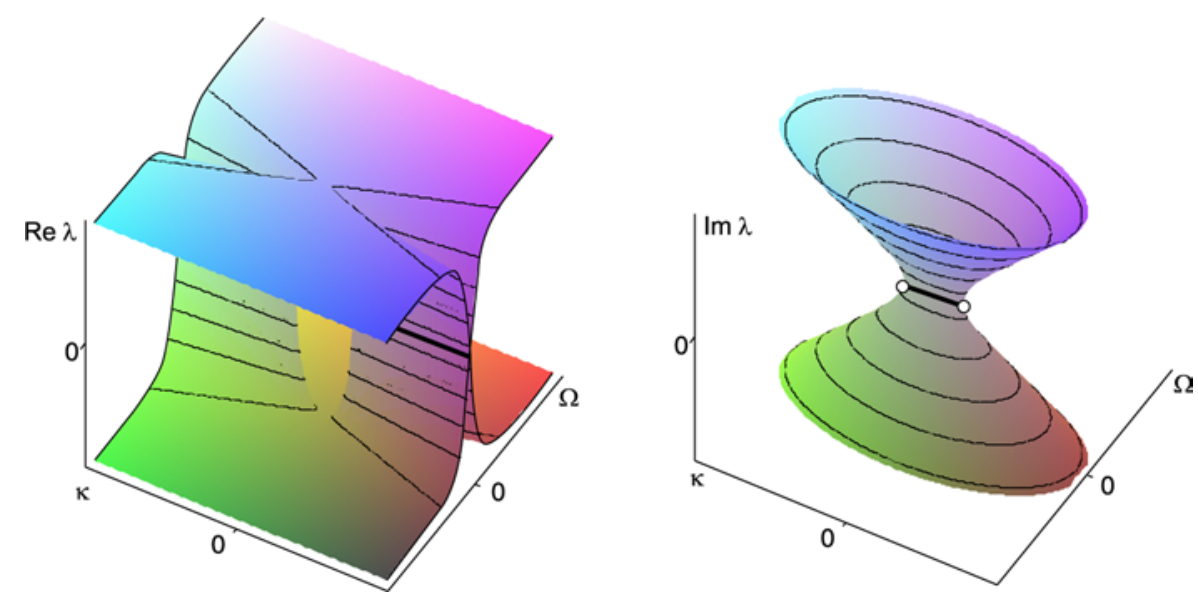

\section{Conclusion}

Stability of a symmetric rotor in contact with the friction pads is sensitive to the structural modifications of the latter. From the other hand, the relative simplicity of the unperturbed symmetric system allows us to develop an efficient sensitivity analysis of the eigenvalues and the critical values of parameters with respect to the stator design variations. This reveals the complex geometry of the stability domain which is determined, however, by a finite number of well known singularities such as conical points and Whitney's umbrellas. Therefore, the complex behaviour of eigenvalues can be efficiently dealt with using the sensitivity analysis developed in the present work. The results obtained illuminate the perspectives and difficulties for structural optimisation of the elements of brakes.

\section{Acknowledgements}

The work has been supported by the research grant DFG HA 1060/43-1. 


\section{References}

Abu Bakar, A.R., Ouyang, H., James, S. and Li, L. (2008) 'Finite element analysis of wear and its effect on squeal generation', IMechE Journal of Automobile Engineering, Vol. 222, No. 7, pp.1153-1165.

Bottema, O. (1956) 'The Routh-Hurwitz condition for the biquadratic equation', Indagationes Mathematicae, Vol. 18, pp.403-406.

Campbell, W. (1924) 'The protection of steam-turbine disk wheels from axial vibration', Transactions of the ASME, Vol. 46, pp.31-160.

Chen, F., Tan, C.A. and Quaglia, R.L. (Eds.) (2006) Disc Brake Squeal: Mechanism, Analysis, Evaluation and Reduction/Prevention, SAE, Warrendale, pp.1-401.

Chevillot, F., Sinou, J-J., Mazet, G-B., Hardouin, N. and Jézéquel, L. (2008) 'The destabilization paradox applied to friction-induced vibrations in an aircraft braking system', Archive of Applied Mechanics, Vol. 78, pp.949-963.

Dobson, I., Zhang, J., Greene, S., Engdahl, H. and Sauer, P.W. (2001) 'Is strong modal resonance a precursor to power system oscillations?', IEEE Trans. Circuits Syst. I, Vol. 48, pp.340-349.

Fieldhouse, J. and Beveridge, C. (2000) 'Comparison of disc and drum brake rotor mode movement', Seoul 2000 FISITA World Automotive Congress, 12-15 June, Seoul, Korea, paper F2000H241.

Fieldhouse, J.D., Steel, W.P., Talbot, C.J. and Siddiqui, M.A. (2004) 'Rotor asymmetry used to reduce disc brake noise', Brake Colloquium and Exhibition, October, Anaheim, CA, USA, SAE, Warrendale, SAE Paper 2004-01-2797.

Fieldhouse, J.D., Ashraf, N. and Talbot, C. (2008) 'The measurement and analysis of the $\mathrm{disc} / \mathrm{pad}$ interface dynamic centre of pressure and its influence on brake noise', $S P-2188$, Brake Technology, SAE, Warrendale, SAE Paper 2008-01-0826.

Gabrielson, T.B. (1999) 'Frequency constants for transverse vibration of annular disks', Journal of the Acoustical Society of America, Vol. 105, No. 6, pp.3311-3317.

Genta, G. (2007) Dynamics of Rotating Systems, Springer, New York, pp.1-658.

Hagedorn, P. (1988) 'Neuere Entwicklungen in der technischen Schwingungslehre', Zeitschrift Für Angewandte Mathematik Und Mechanik, Vol. 68, pp.22-31.

Hoffmann, N.P. and Gaul, L. (2008) 'Friction induced vibrations of brakes: research fields and activities', Brake Colloquium and Exhibition, October, San Antonio, TX, USA, Session: Friction-Induced Vibration, SAE, Warrendale, SAE Paper 2008-01-2584.

Jones, C.A. (1988) 'Multiple eigenvalues and mode classification in plane Poiseuille flow', The Quarterly Journal of Mechanics and Applied Mathematics, Vol. 41, No. 3, pp.363-382.

Kang, J., Krousgrill, C.M. and Sadeghi, F. (2008) 'Dynamic instability of a thin circular plate with friction interface and its application to disc brake squeal', Journal of Sound and Vibration, Vol. 316, Nos. 1-5, pp.164-179.

Kinkaid, N.M., O'Reilly, O.M. and Papadopoulos, P. (2003) 'Automotive disc brake squeal', Journal of Sound and Vibration, Vol. 267, pp.105-166.

Kirillov, O.N. and Seyranian, A.P. (1998) 'Optimization of stability of a flexible missile under follower thrust', 7th AIAA/USAF/NASA/ISSMO Symposium on Multidisciplinary Analysis and Optimization, 2-4 September, St. Louis, Missouri, USA, pp.2063-2073, AIAA Paper 98-4969.

Kirillov, O.N., Mailybaev, A.A. and Seyranian, A.P. (2005) 'Unfolding of eigenvalue surfaces near a diabolic point due to a complex perturbation', Journal of Physics A: Mathematical and General, Vol. 38, No. 24. pp.5531-5546.

Kirillov, O.N. (2007a) 'Destabilization paradox due to breaking the Hamiltonian and reversible symmetry', International Journal of Non-Linear Mechanics, Vol. 42, No. 1, pp.71-87. 
Kirillov, O.N. (2007b) 'Gyroscopic stabilization in the presence of nonconservative forces', Doklady Mathematics, Vol. 76, No. 2, pp.780-785.

Kirillov, O.N. (2008a) 'How to play a disc brake: a dissipation-induced squeal', Noise and Vibration, SP-2158, SAE World Congress and Exhibition, 14-17 April, Detroit, MI, USA, pp.167-180, SAE Paper 2008-01-1160.

Kirillov, O.N. (2008b) 'Subcritical flutter in the acoustics of friction', Proceedings of the Royal Society A, Vol. 464, No. 2097, pp.2321-2339.

Lakhadanov, V.M. (1975) 'On stabilization of potential systems', Prikladnaya Matematika i Mekhanika, Vol. 39, pp.53-58.

Lee, C-W., Han, D-J., Suh, J-H. and Hong, S-W. (2007) 'Modal analysis of periodically time-varying linear rotor systems', Journal of Sound and Vibration, Vol. 303, pp.553-574.

MacKay, R.S. (1986) 'Stability of equilibria of Hamiltonian systems', in Sarkar, S. (Ed.): Nonlinear Phenomena and Chaos, Adam Hilger, Bristol, UK, pp.254-270.

Mottershead, J.E. (1998) 'Vibration- and friction-induced instabilities in discs', The Shock and Vibration Digest, Vol. 30, pp.14-31.

Ostermeyer, G.P. and Müller, M. (2008) 'New insights into the tribology of brake systems', IMechE Journal of Automobile Engineering, Vol. 222, pp.1167-1200.

Oura, Y., Kurita, Y., Matsumura, Y. and Nishizawa, Y. (2008) 'Influence of distributed stiffness in contact surface on disk brake squeal', Brake Colloquium and Exhibition, October, San Antonio, TX, USA, Session: Friction-Induced Vibration, SAE, Warrendale, SAE Paper 2008-01-2584.

Ouyang, H., Nack, W., Yuan, Y. and Chen, F. (2005) 'Numerical analysis of automotive disc brake squeal: a review', International Journal of Vehicle Noise and Vibration, Vol. 1, Nos. 3-4, pp.207-231.

Ouyang, H. (2008) 'Prediction and assignment of latent roots of damped asymmetric systems by structural modifications', Mechanical Systems and Signal Processing, DOI: $10.1016 /$ j.ymssp.2008.08.001

Penninger, C.L. and Swift, R.A. (2004) 'Disc brake lining shape optimization by multibody dynamic analysis', SP-1866, ABS/TCS, Brake Technology and Foundation Brake NVH, and Tire and Wheel Technology, SAE, Warrendale, SAE Paper 2004-01-0725.

Seyranian, A.P., Lund, E. and Olhoff, N. (1994) 'Multiple-eigenvalues in structural optimization problems', Structural Optimization, Vol. 8, No. 4, pp.207-227.

Spurr, R.T. (1961) 'The ringing of wine glasses', Wear, Vol. 4, pp.150-153.

Spelsberg-Korspeter, G., Hochlenert, D., Kirillov, O.N. and Hagedorn, P. (2009) 'In- and out-of-plane vibrations of a rotating plate with frictional contact: investigations on squeal phenomena', Trans. ASME, J. Appl. Mech., Vol. 76, No. 4, p.041006.

Xiong, L.G., Chen, H. and Yi, J.M. (2002) 'Instability mechanism of a rotating disc subjected to various transverse interactive forces', Journal of Materials Processing Technology, Vol. 129, pp.534-538.

Xiong, Y. and Hutton, S. (1994) 'Vibration and stability analysis of a multy-guided rotating string', Journal of Sound and Vibration, Vol. 169, No. 5, pp.669-683.

Yang, L. and Hutton, S.G. (1995) 'Interactions between an idealized rotating string and stationary constraints', Journal of Sound and Vibration, Vol. 185, pp.139-154. 